\title{
INDUCTIVE FREENESS OF ZIEGLER'S CANONICAL MULTIDERIVATIONS FOR REFLECTION ARRANGEMENTS
}

\author{
TORSTEN HOGE AND GERHARD RÖHRLE
}

\begin{abstract}
Let $\mathscr{A}$ be a free hyperplane arrangement. In 1989, Ziegler showed that the restriction $\mathscr{A}^{\prime \prime}$ of $\mathscr{A}$ to any hyperplane endowed with the natural multiplicity is then a free multiarrangement. We initiate a study of the stronger freeness property of inductive freeness for these canonical free multiarrangements and investigate them for the underlying class of reflection arrangements.

More precisely, let $\mathscr{A}=\mathscr{A}(W)$ be the reflection arrangement of a complex reflection group $W$. By work of Terao, each such reflection arrangement is free. Thus so is Ziegler's canonical multiplicity on the restriction $\mathscr{A}^{\prime \prime}$ of $\mathscr{A}$ to a hyperplane. We show that the latter is inductively free as a multiarrangement if and only if $\mathscr{A}^{\prime \prime}$ itself is inductively free.
\end{abstract}

\section{Contents}

1. Introduction 1

2. Recollections and Preliminaries 4

3. Filtrations of Free Multiplicities 11

4. Proofs of Theorems 1.4 and $1.9 \quad 14$

References 22

\section{INTRODUCTION}

The class of free arrangements, respectively free multiarrangments, plays a fundamental role in the theory of hyperplane arrangements, respectively multiarrangements. In his seminal work [Z89], Ziegler introduced the notion of multiarrangements and initiated the study of their freeness. We begin by recalling Ziegler's fundamental construction from loc. cit.

Definition 1.1. Let $\mathscr{A}$ be an arrangement. Fix $H_{0} \in \mathscr{A}$ and consider the restriction $\mathscr{A}^{\prime \prime}$ with respect to $H_{0}$. Define the canonical multiplicity $\kappa$ on $\mathscr{A}^{\prime \prime}$ as follows. For $Y \in \mathscr{A}^{\prime \prime}$ set

$$
\kappa(Y):=\left|\mathscr{A}_{Y}\right|-1,
$$

i.e., $\kappa(Y)$ is the number of hyperplanes in $\mathscr{A} \backslash\left\{H_{0}\right\}$ lying above $Y$. Ziegler showed that freeness of $\mathscr{A}$ implies freeness of $\left(\mathscr{A}^{\prime \prime}, \kappa\right)$ as follows.

2010 Mathematics Subject Classification. Primary 20F55, 51F15, 52C35, 14N20, 32S22; Secondary 51D20.

Key words and phrases. Multiarrangement, Ziegler multiplicity, free arrangement, inductively free arrangement, reflection arrangement. 
Theorem 1.2 ([Z89, Thm. 11]). Let $\mathscr{A}$ be a free arrangement with exponents $\exp \mathscr{A}=$ $\left\{1, e_{2}, \ldots, e_{\ell}\right\}$. Let $H_{0} \in \mathscr{A}$ and consider the restriction $\mathscr{A}^{\prime \prime}$ with respect to $H_{0}$. Then the multiarrangement $\left(\mathscr{A}^{\prime \prime}, \kappa\right)$ is free with exponents $\exp \left(\mathscr{A}^{\prime \prime}, \kappa\right)=\left\{e_{2}, \ldots, e_{\ell}\right\}$.

Note that the converse of Theorem 1.2 is false. For let $\mathscr{A}$ be a non-free 3 -arrangement, cf. [OT92, Ex. 4.34]. Since $\mathscr{A}^{\prime \prime}$ is of rank 2, $\left(\mathscr{A}^{\prime \prime}, \kappa\right)$ is free, [Z89, Cor. 7]. Nevertheless, Ziegler's construction and in particular the question of a converse of Theorem 1.2 under suitable additional hypotheses play an important role in the study of free simple arrangements, [Y04, Thms. 2.1, 2.2], [Y05], [AY13, Cor. 4.2], [S12, Thm. 2] and [Y14, Cor. 1.35].

Because of the relevance of Ziegler's multiplicity in the theory of free arrangements, it is natural to investigate stronger freeness properties for $\left(\mathscr{A}^{\prime \prime}, \kappa\right)$ and specifically to ask for an analogue of Theorem 1.2 for inductive freeness.

Question 1.3. Is it the case that $\left(\mathscr{A}^{\prime \prime}, \kappa\right)$ is inductively free whenever $\mathscr{A}$ is?

In this paper we initialize the study of this question and examine the multiarrangements $\left(\mathscr{A}^{\prime \prime}, \kappa\right)$ where the underlying class consists of reflection arrangements. It turns out that for this class the answer is "yes". While inductive freeness is a combinatorial property for simple arrangements, i.e. it only depends on the underlying intersection lattice, in contrast, for multiarrangements it is not combinatorial in general, [Z89, Prop. 10]. So one might not expect the implication in Question 1.3 to hold for general arrangements $\mathscr{A}$. However, having extensively investigated many examples, we have been unable to come across a counterexample. So it is quite likely that this implication does indeed always hold.

It can be quite cumbersome to decide whether a given (multi)arrangement is inductively free, see for instance [BC12, §5.2], [AHR14, Lem. 4.2], [HR15, Lem. 3.5], [CR16, Thm. 1.1] and [CRS17, Thm. 1.4]. This might entail an extensive perusal of a large number of chains of free subarrangements.

If $\mathscr{A}=\mathscr{A}(W)$ is a reflection arrangement of the complex reflection group $W$, then $\mathscr{A}$ is free, thanks to work of Terao [T80b]. Thus $\left(\mathscr{A}^{\prime \prime}, \kappa\right)$ is also free, by Theorem 1.2.

In our main result, we classify all instances when Ziegler's canonical multiplicity $\left(\mathscr{A}^{\prime \prime}, \kappa\right)$ is inductively free in case the underlying arrangement $\mathscr{A}$ is a reflection arrangement. In particular, we answer Question 1.3 in the affirmative for this class of arrangements.

Because of the compatibility of the product constructions for inductive freeness for simple arrangements, [HR15, Prop. 2.10], as well as for multiarrangements, [HRS17, Thm. 1.4] (cf. Theorem 2.23), the question of inductive freeness of $\left(\mathscr{A}^{\prime \prime}, \kappa\right)$ readily reduces to the case when $\mathscr{A}$ is irreducible. Thus for a reflection arrangement $\mathscr{A}(W)$, we may assume that $W$ is irreducible.

Theorem 1.4. Let $\mathscr{A}=\mathscr{A}(W)$ be the reflection arrangement of the irreducible complex reflection group $W$. Let $\mathscr{A}^{\prime \prime}$ be the restriction of $\mathscr{A}$ to a hyperplane in $\mathscr{A}$. Then $\left(\mathscr{A}^{\prime \prime}, \kappa\right)$ is inductively free if and only if one of the following holds:

(i) $\mathscr{A}$ is inductively free; or

(ii) $\mathscr{A}$ is non-inductively free of rank at most 4 . 
We record several consequences of Theorem 1.4. Our first observation follows from the theorem along with the classification of all inductively free restrictions of reflection arrangements from [AHR14, Thm. 1.2], see Theorem 2.4 below.

Corollary 1.5. Let $\mathscr{A}=\mathscr{A}(W)$ be the reflection arrangement of the complex reflection group $W$. Then $\left(\mathscr{A}^{\prime \prime}, \kappa\right)$ is inductively free if and only if $\mathscr{A}^{\prime \prime}$ itself is inductively free.

We mention that Corollary 1.5 is false in general. For the failure of the forward implication, see [HR15, Ex. 2.16]. There we present an example of an inductively free arrangement $\mathscr{A}$ where a particular restriction to a hyperplane $\mathscr{A}^{\prime \prime}$ fails to be free. In particular, $\mathscr{A}^{\prime \prime}$ is not inductively free. Nevertheless, since $\mathscr{A}$ is free, so is $\left(\mathscr{A}^{\prime \prime}, \kappa\right)$, thanks to Theorem 1.2. One can check that in this instance $\left(\mathscr{A}^{\prime \prime}, \kappa\right)$ is actually inductively free. Nevertheless, this example is consistent with the assertion in Question 1.3. Example 2.24 shows that the reverse implication in Corollary 1.5 also fails in general. In view of these elementary examples, the equivalence of Corollary 1.5 is rather striking and underlines the very special nature of reflection arrangements.

In addition to the canonical multiplicity from Definition 1.1, we study multiplicities which are concentrated at a single hyperplane. These were introduced by Abe, Terao and Wakefield, $[$ ATW08, §5]. While in general, $(\mathscr{A}, \mu)$ need not be free for a free hyperplane arrangement $\mathscr{A}$ and an arbitrary multiplicity $\mu$, e.g. see [Z89, Ex. 14], for these concentrated multiplicities freeness is also inherited from the simple arrangement. It turns out that they are closely related to Ziegler's canonical multiplicity, see Propositions 2.14 and 2.15.

Definition 1.6. Let $\mathscr{A}$ be a simple arrangement. Fix $H_{0} \in \mathscr{A}$ and $m_{0} \in \mathbb{Z}_{\geq 1}$ and define the multiplicity $\delta$ concentrated at $H_{0}$ by

$$
\delta(H):=\delta_{H_{0}, m_{0}}(H):= \begin{cases}m_{0} & \text { if } H=H_{0} \\ 1 & \text { else. }\end{cases}
$$

It turns out that in this instance, both $\mathscr{A}$ and $(\mathscr{A}, \delta)$ inherit freeness from each other, as opposed to the general case indicated above. We record this in our next result.

Theorem 1.7. Let $\mathscr{A}$ be an arrangement. Fix $H_{0} \in \mathscr{A}, m_{0} \in \mathbb{Z}_{\geq 1}$ and let $\delta=\delta_{H_{0}, m_{0}}$ be the multiplicity concentrated at $H_{0}$, as in Definition 1.6. Then $\mathscr{A}$ is free with exponents $\exp \mathscr{A}=$ $\left\{1, e_{2}, \ldots, e_{\ell}\right\}$ if and only if $(\mathscr{A}, \delta)$ is free with exponents $\exp (\mathscr{A}, \delta)=\left\{m_{0}, e_{2}, \ldots, e_{\ell}\right\}$.

The forward implication of Theorem 1.7 is [ATW08, Prop. 5.2] and the reverse implication is Proposition 2.15(i).

As in the case of Ziegler's canonical multiplicity, one might also speculate whether the analogue for inductive freeness holds for the forward implication of Theorem 1.7.

Question 1.8. Is it the case that $(\mathscr{A}, \delta)$ is inductively free whenever $\mathscr{A}$ is?

It turns out that Questions 1.3 and 1.8 are closely related: the assertion of Question 1.8 follows from that in Question 1.3, see Corollary 2.20.

Let $\mathscr{A}=\mathscr{A}(W)$ be the reflection arrangement of the complex reflection group $W$. Since $\mathscr{A}$ is free, it follows from Proposition 2.14(i) that the multiarrangement $(\mathscr{A}, \delta)$ is also free. In 
[HR15, Thm. 1.1], we classified all inductively free reflection arrangements. In our second main result we extend this classification to multiarrangements of the form $(\mathscr{A}(W), \delta)$. This in particular gives an affirmative answer to Question 1.8 for the class of reflection arrangements.

Theorem 1.9. Let $\mathscr{A}=\mathscr{A}(W)$ be the reflection arrangement of the complex reflection group $W$. Then $(\mathscr{A}, \delta)$ is inductively free if and only if $\mathscr{A}$ is inductively free.

The paper is organized as follows. In Section 2.2 we recall the fundamental results for free arrangements, in particular Terao's Addition Deletion Theorem 2.1 and the subsequent notion of an inductively free arrangement. The classification of the inductively free reflection arrangements from [HR15, Thm. 1.1], and the classification of the inductively free restrictions of reflection arrangements from [AHR14, Thm. 1.2] are restated in Section 2.3. Section 2.5 is devoted to multiarrangements and their freeness. Here we present the Addition Deletion theorem due to Abe, Terao, and Wakefield, [ATW08, Thm. 0.8]. In Section 2.6 we treat Ziegler's canonical and concentrated multiplicities. This is followed by a discussion of inductive freeness for multiarrangements in Section 2.7. Here we recall results from [HRS17] which show the compatibility of this notion with products and localization for multiarrangements that are used in the sequel.

Section 3 is central in the paper. Here we prove some general results for free arrangements which show that under weak conditions on the exponents of a free arrangement $\mathscr{A}$ and a given free restriction $\mathscr{A}^{H}$, every multiplicity between the simple and the Ziegler multiplicity on $\mathscr{A}^{H}$ is already free, see Corollary 3.9. This is then applied to reflection arrangements in Proposition 3.10. We then apply these methods to the imprimitive reflection groups $G(r, 1, \ell-1)$. Combined with the fact that the reflection arrangement $\mathscr{A}(G(r, 1, \ell-1))$ itself is inductively free (cf. Theorem 2.3), we show that $\left(\mathscr{A}(G(r, 1, \ell))^{\prime \prime}, \kappa\right)$ is inductively free in Proposition 3.11. As a further relevant consequence for our classification, we derive that $\left(\mathscr{A}(W)^{\prime \prime}, \kappa\right)$ is inductively free, for $W$ of type $F_{4}, H_{4}, G_{31}$ and $G_{32}$ in Corollary 3.14.

In Section 4 we present our proofs of Theorems 1.4 and 1.9. The fact that inductively free multiarrangements are closed under localization proves extremely useful in this context, see Theorem 2.22. By this means, we can for instance deduce that $\left(\mathscr{A}\left(E_{6}\right)^{\prime \prime}, \kappa\right)$ and $\left(\mathscr{A}\left(E_{7}\right)^{\prime \prime}, \kappa\right)$ are inductively free from the fact that $\left(\mathscr{A}\left(E_{8}\right)^{\prime \prime}, \kappa\right)$ is so, see Lemma 4.5. The same theorem in turn allows us to derive that $\left(\mathscr{A}\left(G_{34}\right)^{\prime \prime}, \kappa\right)$ is not inductively free from the fact that $\left(\mathscr{A}(G(3,3,5))^{\prime \prime}, \kappa\right)$ isn't either, see Lemma 4.10 .

Due to its large rank, the proof of inductive freeness for $\left(\mathscr{A}\left(E_{8}\right)^{\prime \prime}, \kappa\right)$ is the most challenging among all groups of exceptional type. It involves delicate arguments of induction, see Lemma 4.5. A crucial ingredient in our proof is the fact that on $\mathscr{A}\left(E_{8}\right)^{\prime \prime}$ every multiplicity between the simple one and Ziegler's multiplicity $\kappa$ is free, thus readily providing free filtrations of $\left(\mathscr{A}\left(E_{8}\right)^{\prime \prime}, \kappa\right)$ for our analysis, see Proposition 3.10.

\section{Recollections And Preliminaries}

2.1. Hyperplane Arrangements. Let $V=\mathbb{K}^{\ell}$ be an $\ell$-dimensional $\mathbb{K}$-vector space. A hyperplane arrangement is a pair $(\mathscr{A}, V)$, where $\mathscr{A}$ is a finite collection of hyperplanes in $V$. 
Usually, we simply write $\mathscr{A}$ in place of $(\mathscr{A}, V)$. We write $|\mathscr{A}|$ for the number of hyperplanes in $\mathscr{A}$. The empty arrangement in $V$ is denoted by $\Phi_{\ell}$.

The lattice $L(\mathscr{A})$ of $\mathscr{A}$ is the set of subspaces of $V$ of the form $H_{1} \cap \cdots \cap H_{i}$ where $\left\{H_{1}, \ldots, H_{i}\right\}$ is a subset of $\mathscr{A}$. For $X \in L(\mathscr{A})$, we have two associated arrangements, firstly $\mathscr{A}_{X}:=\{H \in$ $\mathscr{A} \mid X \subseteq H\} \subseteq \mathscr{A}$, the localization of $\mathscr{A}$ at $X$, and secondly, the restriction of $\mathscr{A}$ to $X$, $\left(\mathscr{A}^{X}, X\right)$, where $\mathscr{A}^{X}:=\left\{X \cap H \mid H \in \mathscr{A} \backslash \mathscr{A}_{X}\right\}$. Note that $V$ belongs to $L(\mathscr{A})$ as the intersection of the empty collection of hyperplanes and $\mathscr{A}^{V}=\mathscr{A}$. The lattice $L(\mathscr{A})$ is a partially ordered set by reverse inclusion: $X \leq Y$ provided $Y \subseteq X$ for $X, Y \in L(\mathscr{A})$.

If $0 \in H$ for each $H$ in $\mathscr{A}$, then $\mathscr{A}$ is called central. If $\mathscr{A}$ is central, then the center $T_{\mathscr{A}}:=\cap_{H \in \mathscr{A}} H$ of $\mathscr{A}$ is the unique maximal element in $L(\mathscr{A})$ with respect to the partial order. We have a rank function on $L(\mathscr{A}): r(X):=\operatorname{codim}_{V}(X)$. The rank $r:=r(\mathscr{A})$ of $\mathscr{A}$ is the rank of a maximal element in $L(\mathscr{A})$. The $\ell$-arrangement $\mathscr{A}$ is essential provided $r(\mathscr{A})=\ell$. If $\mathscr{A}$ is central and essential, then $T_{\mathscr{A}}=\{0\}$. Throughout, we only consider central arrangements.

2.2. Free Hyperplane Arrangements. Let $S=S\left(V^{*}\right)$ be the symmetric algebra of the dual space $V^{*}$ of $V$. If $x_{1}, \ldots, x_{\ell}$ is a basis of $V^{*}$, then we identify $S$ with the polynomial ring $\mathbb{K}\left[x_{1}, \ldots, x_{\ell}\right]$. Letting $S_{p}$ denote the $\mathbb{K}$-subspace of $S$ consisting of the homogeneous polynomials of degree $p$ (along with 0 ), $S$ is naturally $\mathbb{Z}$-graded: $S=\oplus_{p \in \mathbb{Z}} S_{p}$, where $S_{p}=0$ in case $p<0$.

Let $\operatorname{Der}(S)$ be the $S$-module of algebraic $\mathbb{K}$-derivations of $S$. Using the $\mathbb{Z}$-grading on $S$, $\operatorname{Der}(S)$ becomes a graded $S$-module. For $i=1, \ldots, \ell$, let $D_{i}:=\partial / \partial x_{i}$ be the usual derivation of $S$. Then $D_{1}, \ldots, D_{\ell}$ is an $S$-basis of $\operatorname{Der}(S)$. We say that $\theta \in \operatorname{Der}(S)$ is homogeneous of polynomial degree $p$ provided $\theta=\sum_{i=1}^{\ell} f_{i} D_{i}$, where $f_{i}$ is either 0 or homogeneous of degree $p$ for each $1 \leq i \leq \ell$. In this case we write $\operatorname{pdeg} \theta=p$.

Let $\mathscr{A}$ be an arrangement in $V$. Then for $H \in \mathscr{A}$ we fix $\alpha_{H} \in V^{*}$ with $H=\operatorname{ker}\left(\alpha_{H}\right)$. The defining polynomial $Q(\mathscr{A})$ of $\mathscr{A}$ is given by $Q(\mathscr{A}):=\prod_{H \in \mathscr{A}} \alpha_{H} \in S$.

The module of $\mathscr{A}$-derivations of $\mathscr{A}$ is defined by

$$
D(\mathscr{A}):=\left\{\theta \in \operatorname{Der}(S) \mid \theta\left(\alpha_{H}\right) \in \alpha_{H} S \text { for each } H \in \mathscr{A}\right\} .
$$

We say that $\mathscr{A}$ is free if the module of $\mathscr{A}$-derivations $D(\mathscr{A})$ is a free $S$-module.

With the $\mathbb{Z}$-grading of $\operatorname{Der}(S)$, also $D(\mathscr{A})$ becomes a graded $S$-module, [OT92, Prop. 4.10]. If $\mathscr{A}$ is a free arrangement, then the $S$-module $D(\mathscr{A})$ admits a basis of $\ell$ homogeneous derivations, say $\theta_{1}, \ldots, \theta_{\ell}$, [OT92, Prop. 4.18]. While the $\theta_{i}$ 's are not unique, their polynomial degrees pdeg $\theta_{i}$ are unique (up to ordering). This multiset is the set of exponents of the free arrangement $\mathscr{A}$ and is denoted by $\exp \mathscr{A}$.

The fundamental Addition Deletion Theorem due to Terao [T80a] plays a crucial role in the study of free arrangements, [OT92, Thm. 4.51].

Theorem 2.1. Suppose $\mathscr{A} \neq \Phi_{\ell}$ and let $\left(\mathscr{A}, \mathscr{A}^{\prime}, \mathscr{A}^{\prime \prime}\right)$ be a triple of arrangements. Then any two of the following statements imply the third:

(i) $\mathscr{A}$ is free with $\exp \mathscr{A}=\left\{b_{1}, \ldots, b_{\ell-1}, b_{\ell}\right\}$; 
(ii) $\mathscr{A}^{\prime}$ is free with $\exp \mathscr{A}^{\prime}=\left\{b_{1}, \ldots, b_{\ell-1}, b_{\ell}-1\right\}$;

(iii) $\mathscr{A}^{\prime \prime}$ is free with $\exp \mathscr{A}^{\prime \prime}=\left\{b_{1}, \ldots, b_{\ell-1}\right\}$.

Theorem 2.1 motivates the notion of inductively free arrangements, cf. [OT92, Def. 4.53]:

Definition 2.2. The class $\mathcal{I F}$ of inductively free arrangements is the smallest class of arrangements subject to

(i) $\Phi_{\ell} \in \mathcal{I F}$ for each $\ell \geq 0$;

(ii) if there exists a hyperplane $H_{0} \in \mathscr{A}$ such that both $\mathscr{A}^{\prime}$ and $\mathscr{A}^{\prime \prime}$ belong to $\mathcal{I F}$, and $\exp \mathscr{A}^{\prime \prime} \subseteq \exp \mathscr{A}^{\prime}$, then $\mathscr{A}$ also belongs to $\mathcal{I F}$.

2.3. Reflection Arrangements. The irreducible finite complex reflection groups were classified by Shephard and Todd, [ST54]. Let $W \subseteq \mathrm{GL}(V)$ be a finite complex reflection group. For $w \in W$, we write $\operatorname{Fix}(w):=\{v \in V \mid w v=v\}$ for the fixed point subspace of $w$. For $U \subseteq V$ a subspace, we define the parabolic subgroup $W_{U}$ of $W$ by $W_{U}:=\{w \in W \mid U \subseteq$ $\operatorname{Fix}(w)\}$.

The reflection arrangement $\mathscr{A}=\mathscr{A}(W)$ of $W$ in $V$ is the hyperplane arrangement consisting of the reflecting hyperplanes of the elements in $W$ acting as reflections on $V$. By Steinberg's Theorem [Ste60, Thm. 1.5], for $X \in L(\mathscr{A})$, the parabolic subgroup $W_{X}$ is itself a complex reflection group, generated by the unitary reflections in $W$ that are contained in $W_{X}$. Thus, we identify the reflection arrangement $\mathscr{A}\left(W_{X}\right)$ of $W_{X}$ as a subarrangement of $\mathscr{A}$.

Using the classification and nomenclature of Shephard and Todd [ST54], we recall the main classification results from [HR15, Thm. 1.1] and [AHR14, Thm. 1.2], respectively.

Theorem 2.3. For a finite complex reflection group $W$, its reflection arrangement $\mathscr{A}(W)$ is inductively free if and only if $W$ does not admit an irreducible factor isomorphic to a monomial group $G(r, r, \ell)$ for $r, \ell \geq 3, G_{24}, G_{27}, G_{29}, G_{31}, G_{33}$, or $G_{34}$.

Theorem 2.4. Let $W$ be a finite, irreducible, complex reflection group with reflection arrangement $\mathscr{A}=\mathscr{A}(W)$ and let $X \in L(\mathscr{A})$. The restricted arrangement $\mathscr{A}^{X}$ is inductively free if and only if one of the following holds:

(i) $\mathscr{A}$ is inductively free;

(ii) $W=G(r, r, \ell)$ and $\mathscr{A}^{X} \cong \mathscr{A}_{p}^{k}(r)$, where $p=\operatorname{dim} X$ and $p-2 \leq k \leq p$;

(iii) $W$ is one of $G_{24}, G_{27}, G_{29}, G_{31}, G_{33}$, or $G_{34}$ and $X \in L(\mathscr{A}) \backslash\{V\}$ with $\operatorname{dim} X \leq 3$.

2.4. Multiarrangements. A multiarrangement is a pair $(\mathscr{A}, \mu)$ consisting of a hyperplane arrangement $\mathscr{A}$ and a multiplicity function $\mu: \mathscr{A} \rightarrow \mathbb{Z}_{\geq 0}$ associating to each hyperplane $H$ in $\mathscr{A}$ a non-negative integer $\mu(H)$. Alternately, the multiarrangement $(\mathscr{A}, \mu)$ can also be thought of as the multiset of hyperplanes

$$
(\mathscr{A}, \mu)=\left\{H^{\mu(H)} \mid H \in \mathscr{A}\right\} .
$$

The order of the multiarrangement $(\mathscr{A}, \mu)$ is the cardinality of the multiset $(\mathscr{A}, \mu)$; we write $|\mu|:=|(\mathscr{A}, \mu)|=\sum_{H \in \mathscr{A}} \mu(H)$. For a multiarrangement $(\mathscr{A}, \mu)$, the underlying arrangement 
$\mathscr{A}$ is sometimes called the associated simple arrangement, and so $(\mathscr{A}, \mu)$ itself is simple if and only if $\mu(H)=1$ for each $H \in \mathscr{A}$.

Let $(\mathscr{A}, \mu)$ be a multiarrangement in $V$ and let $X \in L(\mathscr{A})$. The localization of $(\mathscr{A}, \mu)$ at $X$ is defined to be $\left(\mathscr{A}_{X}, \mu_{X}\right)$, where $\mu_{X}=\left.\mu\right|_{\mathscr{A}_{X}}$.

There is a natural partial order on the set of multiplicities for a given simple arrangement, as follows.

Definition 2.5. For multiplicities $\mu_{1}$ and $\mu_{2}$ on an arrangement $\mathscr{A}$, we define $\mu_{1} \leq \mu_{2}$ provided $\mu_{1}(H) \leq \mu_{2}(H)$ for every $H$ in $\mathscr{A}$.

2.5. Freeness of multiarrangements. Following Ziegler [Z89], we extend the notion of freeness to multiarrangements as follows. The defining polynomial $Q(\mathscr{A}, \mu)$ of the multiarrangement $(\mathscr{A}, \mu)$ is given by

$$
Q(\mathscr{A}, \mu):=\prod_{H \in \mathscr{A}} \alpha_{H}^{\mu(H)}
$$

a polynomial of degree $|\mu|$ in $S$.

The module of $\mathscr{A}$-derivations of $(\mathscr{A}, \mu)$ is defined by

$$
D(\mathscr{A}, \mu):=\left\{\theta \in \operatorname{Der}(S) \mid \theta\left(\alpha_{H}\right) \in \alpha_{H}^{\mu(H)} S \text { for each } H \in \mathscr{A}\right\} .
$$

We say that $(\mathscr{A}, \mu)$ is free if $D(\mathscr{A}, \mu)$ is a free $S$-module, [Z89, Def. 6].

As in the case of simple arrangements, $D(\mathscr{A}, \mu)$ is a $\mathbb{Z}$-graded $S$-module and thus, if $(\mathscr{A}, \mu)$ is free, there is a homogeneous basis $\theta_{1}, \ldots, \theta_{\ell}$ of $D(\mathscr{A}, \mu)$. The multiset of the unique polynomial degrees pdeg $\theta_{i}$ forms the set of exponents of the free multiarrangement $(\mathscr{A}, \mu)$ and is denoted by $\exp (\mathscr{A}, \mu)$.

Next we recall Ziegler's analogue of Saito's criterion.

Theorem 2.6 ([Z89, Thm. 8]). Let $\theta_{1}, \ldots, \theta_{\ell}$ be in $D(\mathscr{A}, \mu)$. Then $\left\{\theta_{1}, \ldots, \theta_{\ell}\right\}$ is a basis of $D(\mathscr{A}, \mu)$ if and only if

$$
\operatorname{det} M\left(\theta_{1}, \ldots, \theta_{\ell}\right) \doteq Q(\mathscr{A}, \mu) .
$$

In particular, if $\theta_{1}, \ldots, \theta_{\ell}$ are all homogeneous, then $\left\{\theta_{1}, \ldots, \theta_{\ell}\right\}$ is a basis of $D(\mathscr{A}, \mu)$ if and only if $\theta_{1}, \ldots, \theta_{\ell}$ are independent over $S$ and

$$
\sum \operatorname{pdeg} \theta_{i}=\operatorname{deg} Q(\mathscr{A}, \mu)=|\mu| .
$$

Here the notation $\doteq$ indicates equality up to a non-zero scalar multiple and $M\left(\theta_{1}, \ldots, \theta_{\ell}\right)$ is the coefficient matrix of $\left\{\theta_{1}, \ldots, \theta_{\ell}\right\}$, see [OT92, Def. 4.11].

Note that, owing to $(2.7)$, if $(\mathscr{A}, \mu)$ is free, one can recover $\mu$ from the basis $\left\{\theta_{1}, \ldots, \theta_{\ell}\right\}$.

We recall the construction from [ATW08] for the counterpart of Theorem 2.1 in this more general setting.

Definition 2.9. Let $(\mathscr{A}, \mu) \neq \Phi_{\ell}$ be a multiarrangement. Fix $H_{0}$ in $\mathscr{A}$. We define the deletion $\left(\mathscr{A}^{\prime}, \mu^{\prime}\right)$ and restriction $\left(\mathscr{A}^{\prime \prime}, \mu^{*}\right)$ of $(\mathscr{A}, \mu)$ with respect to $H_{0}$ as follows. If $\mu\left(H_{0}\right)=$ 
1, then set $\mathscr{A}^{\prime}=\mathscr{A} \backslash\left\{H_{0}\right\}$ and define $\mu^{\prime}(H)=\mu(H)$ for all $H \in \mathscr{A}^{\prime}$. If $\mu\left(H_{0}\right)>1$, then set $\mathscr{A}^{\prime}=\mathscr{A}$ and define $\mu^{\prime}\left(H_{0}\right)=\mu\left(H_{0}\right)-1$ and $\mu^{\prime}(H)=\mu(H)$ for all $H \neq H_{0}$.

Let $\mathscr{A}^{\prime \prime}=\left\{H \cap H_{0} \mid H \in \mathscr{A} \backslash\left\{H_{0}\right\}\right\}$. The Euler multiplicity $\mu^{*}$ of $\mathscr{A}^{\prime \prime}$ is defined as follows. Let $Y \in \mathscr{A}^{\prime \prime}$. Since the localization $\mathscr{A}_{Y}$ is of rank 2 , the multiarrangement $\left(\mathscr{A}_{Y}, \mu_{Y}\right)$ is free, [Z89, Cor. 7]. According to [ATW08, Prop. 2.1], the module of derivations $D\left(\mathscr{A}_{Y}, \mu_{Y}\right)$ admits a particular homogeneous basis $\left\{\theta_{Y}, \psi_{Y}, D_{3}, \ldots, D_{\ell}\right\}$, such that $\theta_{Y} \notin \alpha_{0} \operatorname{Der}(S)$ and $\psi_{Y} \in \alpha_{0} \operatorname{Der}(S)$, where $H_{0}=\operatorname{ker} \alpha_{0}$. Then on $Y$ the Euler multiplicity $\mu^{*}$ is defined to be $\mu^{*}(Y)=\operatorname{pdeg} \theta_{Y}$.

Often, $(\mathscr{A}, \mu),\left(\mathscr{A}^{\prime}, \mu^{\prime}\right)$ and $\left(\mathscr{A}^{\prime \prime}, \mu^{*}\right)$ is referred to as the triple of $(\mathscr{A}, \mu)$ with respect to $H_{0}$.

We require some core results from [ATW08].

Theorem 2.10 ([ATW08, Thm. 0.4]). Suppose that $(\mathscr{A}, \mu) \neq \Phi_{\ell}$. Fix $H_{0}$ in $\mathscr{A}$. If both $(\mathscr{A}, \mu)$ and $\left(\mathscr{A}^{\prime}, \mu^{\prime}\right)$ are free, then there exists a basis $\left\{\theta_{1}, \ldots, \theta_{\ell}\right\}$ of $D\left(\mathscr{A}^{\prime}, \mu^{\prime}\right)$ such that $\left\{\theta_{1}, \ldots, \alpha_{k} \theta_{k}, \ldots, \theta_{\ell}\right\}$ is a basis of $D(\mathscr{A}, \mu)$ for some $1 \leq k \leq \ell$.

Fix $H_{0}=\operatorname{ker} \alpha_{0}$ in $\mathscr{A}$ and let $\mathscr{A}^{\prime \prime}$ be the restriction with respect to $H_{0}$. Consider the projection $S \rightarrow \bar{S}:=S / \alpha_{0} S, f \mapsto \bar{f}$. Following [ATW08], there is a canonical map $D(\mathscr{A}, \mu) \rightarrow D\left(\mathscr{A}^{\prime \prime}, \mu^{*}\right), \theta \mapsto \bar{\theta}$, where $\bar{\theta}(\bar{f}):=\overline{\theta(f)}$, for $\bar{f} \in \bar{S}$.

Theorem 2.11 ([ATW08, Thm. 0.6]). Suppose that $(\mathscr{A}, \mu) \neq \Phi_{\ell}$. Fix $H_{0}$ in $\mathscr{A}$. Suppose that both $(\mathscr{A}, \mu)$ and $\left(\mathscr{A}^{\prime}, \mu^{\prime}\right)$ are free. Let $\left\{\theta_{1}, \ldots, \theta_{\ell}\right\}$ be a basis of $D\left(\mathscr{A}^{\prime}, \mu^{\prime}\right)$ as in Theorem 2.10. Then $\left\{\bar{\theta}_{1}, \ldots, \bar{\theta}_{k-1}, \bar{\theta}_{k+1}, \ldots, \bar{\theta}_{\ell}\right\}$ is a basis of $D\left(\mathscr{A}^{\prime \prime}, \mu^{*}\right)$.

Theorem 2.12 ([ATW08, Thm. 0.8] Addition Deletion Theorem for Multiarrangements). Suppose that $(\mathscr{A}, \mu) \neq \Phi_{\ell}$. Fix $H_{0}$ in $\mathscr{A}$ and let $(\mathscr{A}, \mu),\left(\mathscr{A}^{\prime}, \mu^{\prime}\right)$ and $\left(\mathscr{A}^{\prime \prime}, \mu^{*}\right)$ be the triple with respect to $H_{0}$. Then any two of the following statements imply the third:

(i) $(\mathscr{A}, \mu)$ is free with $\exp (\mathscr{A}, \mu)=\left\{b_{1}, \ldots, b_{\ell-1}, b_{\ell}\right\}$;

(ii) $\left(\mathscr{A}^{\prime}, \mu^{\prime}\right)$ is free with $\exp \left(\mathscr{A}^{\prime}, \mu^{\prime}\right)=\left\{b_{1}, \ldots, b_{\ell-1}, b_{\ell}-1\right\}$;

(iii) $\left(\mathscr{A}^{\prime \prime}, \mu^{*}\right)$ is free with $\exp \left(\mathscr{A}^{\prime \prime}, \mu^{*}\right)=\left\{b_{1}, \ldots, b_{\ell-1}\right\}$.

In order to apply Theorem 2.12 effectively, it is crucial to be able to determine the Euler multiplicity for the restrictions at hand. The following is part of [ATW08, Prop. 4.1] relevant for our purposes.

Proposition 2.13. Let $H_{0} \in \mathscr{A}, \mathscr{A}^{\prime \prime}=\mathscr{A}^{H_{0}}$ and let $X \in \mathscr{A}^{\prime \prime}$. Let $\mu$ be a multiplicity on $\mathscr{A}$. Let $\mu_{0}=\mu\left(H_{0}\right)$. Further let $k=\left|\mathscr{A}_{X}\right|$ and $\mu_{1}=\max \left\{\mu(H) \mid H \in \mathscr{A}_{X} \backslash\left\{H_{0}\right\}\right\}$.

(1) If $k=2$, then $\mu^{*}(X)=\mu_{1}$.

(2) If $k=3,2 \mu_{0} \leq\left|\mu_{X}\right|$, and $2 \mu_{1} \leq\left|\mu_{X}\right|$, then $\mu^{*}(X)=\left\lfloor\left|\mu_{X}\right| / 2\right\rfloor$.

(3) If $\left|\mu_{X}\right| \leq 2 k-1$ and $\mu_{0}>0$, then $\mu^{*}(X)=k-1$. 
2.6. Ziegler's Multiplicity and concentrated Multiplicities. Recall Ziegler's multiplicity $\kappa$ from Definition 1.1 and concentrated multiplicities from Definition 1.6. The following combines [ATW08, Prop. 5.2], parts of its proof and Theorem 1.2. The proof of Proposition 2.14(i) given in [ATW08] depends on Theorem 2.12. In [HRS17, Prop. 2.17], we presented an elementary explicit construction for a homogeneous $S$-basis of $D(\mathscr{A}, \delta)$.

Proposition 2.14. Let $\mathscr{A}$ be a free arrangement with exponents $\exp \mathscr{A}=\left\{1, e_{2}, \ldots, e_{\ell}\right\}$. Fix $H_{0} \in \mathscr{A}, m_{0} \in \mathbb{Z}_{\geq 1}$ and let $\delta=\delta_{H_{0}, m_{0}}$ be as in Definition 1.6. Let $\left(\mathscr{A}^{\prime \prime}, \delta^{*}\right)$ be the restriction with respect to $H_{0}$. Then we have

(i) $(\mathscr{A}, \delta)$ is free with exponents $\exp (\mathscr{A}, \delta)=\left\{m_{0}, e_{2}, \ldots, e_{\ell}\right\}$;

(ii) $\left(\mathscr{A}^{\prime \prime}, \delta^{*}\right)=\left(\mathscr{A}^{\prime \prime}, \kappa\right)$ is free with exponents $\exp \left(\mathscr{A}^{\prime \prime}, \kappa\right)=\left\{e_{2}, \ldots, e_{\ell}\right\}$.

Part (i) of the following is a converse of Proposition 2.14(i).

Proposition 2.15. Let $\mathscr{A}$ be an arrangement. Fix $H_{0} \in \mathscr{A}, m_{0} \in \mathbb{Z}_{\geq 1}$ and let $\delta=\delta_{H_{0}, m_{0}}$ be as in Definition 1.6. Let $\left(\mathscr{A}^{\prime \prime}, \delta^{*}\right)$ be the restriction with respect to $\bar{H}_{0}$. Suppose that $(\mathscr{A}, \delta)$ is free with exponents $\exp (\mathscr{A}, \delta)=\left\{m_{0}, e_{2}, \ldots, e_{\ell}\right\}$. Then we have

(i) $\mathscr{A}$ is free with exponents $\exp \mathscr{A}=\left\{1, e_{2}, \ldots, e_{\ell}\right\}$.

(ii) $\left(\mathscr{A}^{\prime \prime}, \delta^{*}\right)=\left(\mathscr{A}^{\prime \prime}, \kappa\right)$ is free with exponents $\exp \left(\mathscr{A}^{\prime \prime}, \kappa\right)=\left\{e_{2}, \ldots, e_{\ell}\right\}$.

Proof. Clearly, part (ii) follows from part (i) and Proposition 2.14(ii).

The proof of (i) is analogous to the one of [OT92, Prop. 4.27]. Let $\alpha_{0} \in V^{*}$ with $H_{0}=\operatorname{ker} \alpha_{0}$. Now let

$$
\operatorname{Ann}\left(H_{0}\right)=\left\{\theta \in D(\mathscr{A}, \delta) \mid \theta\left(\alpha_{0}\right)=0\right\}
$$

be the annihilator of $H_{0}$ in $D(\mathscr{A}, \delta)$ which is a graded $S$-submodule of $D(\mathscr{A}, \delta)$. Let $\theta_{E}$ be the Euler derivation in $\operatorname{Der}(S)$, [OT92, Def. 4.7]. Define

$$
\theta_{\delta}:=\alpha_{0}^{m_{0}-1} \theta_{E} \text {. }
$$

Then $\theta_{\delta}$ belongs to $D(\mathscr{A}, \delta)$. Moreover for each $\theta \in D(\mathscr{A}, \delta)$, we have

$$
\theta-\frac{\theta\left(\alpha_{0}\right)}{\alpha_{0}^{m_{0}}} \theta_{\delta} \in \operatorname{Ann}\left(H_{0}\right) \text {. }
$$

Therefore, since

$$
S \theta_{\delta} \cap \operatorname{Ann}\left(H_{0}\right)=\{0\}
$$

we derive that

$$
D(\mathscr{A}, \delta)=S \theta_{\delta} \oplus \operatorname{Ann}\left(H_{0}\right)
$$

is a direct sum of $S$-modules. Let $\left\{\theta_{2}, \ldots, \theta_{\ell}\right\}$ be a minimal system of homogeneous generators of the $S$-module $\operatorname{Ann}\left(H_{0}\right)$. Then $\left\{\theta_{\delta}, \theta_{2}, \ldots, \theta_{\ell}\right\}$ is a minimal system of homogeneous generators of $D(\mathscr{A}, \delta)$. It follows from [OT92, Thm. A.19], that $\left\{\theta_{\delta}, \theta_{2}, \ldots, \theta_{\ell}\right\}$ is a homogeneous $S$-basis of $D(\mathscr{A}, \delta)$.

Since $\operatorname{Ann}\left(H_{0}\right) \subset D(\mathscr{A}, \delta) \subset D(\mathscr{A})$ are $S$-submodules, $\left\{\theta_{2}, \ldots, \theta_{\ell}\right\}$ is linearly independent over $S$ in $D(\mathscr{A})$. Since $|\delta|=|\mathscr{A}|+m_{0}-1$ and pdeg $\theta_{\delta}=m_{0}$, it follows from Ziegler's analogue of Saito's criterion (3.13) that $\sum_{i=2}^{\ell} \operatorname{pdeg} \theta_{i}=|\mathscr{A}|-1$. Clearly, $\theta_{E}$ belongs to $D(\mathscr{A})$ but not 
to $\operatorname{Ann}\left(H_{0}\right)$. Consequently, it follows from Saito's criterion, [OT92, Thm. 4.23], that $\mathscr{A}$ is free with $\left\{\theta_{E}, \theta_{2}, \ldots, \theta_{\ell}\right\}$ a homogeneous $S$-basis of $D(\mathscr{A})$.

Note that the proof of Proposition 2.15 shows that $m_{0}$ is necessarily an exponent of $(\mathscr{A}, \delta)$ if the latter is free (so it does no harm to require this in the hypothesis).

The following is a special case of a general compatibility result of the Euler multiplicity with localizations from [HRS17, Lem. 2.14].

Lemma 2.16. Let $X \in L(\mathscr{A}), H_{0} \in \mathscr{A}_{X}$, and let $\mathscr{A}^{\prime \prime}$ be the restriction with respect to $H_{0}$. Let $\delta=\delta_{H_{0}, m_{0}}$ be as in Definition 1.6. Then we have

(i) $\left(\left(\mathscr{A}_{X}\right)^{\prime \prime},\left(\delta_{X}\right)^{*}\right)=\left(\left(\mathscr{A}^{\prime \prime}\right)_{X},\left(\delta^{*}\right)_{X}\right)$, and

(ii) $\left(\left(\mathscr{A}_{X}\right)^{\prime \prime}, \kappa\right)=\left(\left(\mathscr{A}^{\prime \prime}\right)_{X}, \kappa_{X}\right)$ (where $\kappa$ on the left is the canonical multiplicity resulting from restriction of $\mathscr{A}_{X}$ to $\left.H_{0}\right)$.

Proof. Part (i) follows from loc. cit. Part (ii) follows from part (i) together with the fact that $\left(\left(\mathscr{A}_{X}\right)^{\prime \prime},\left(\delta_{X}\right)^{*}\right)=\left(\left(\mathscr{A}_{X}\right)^{\prime \prime}, \kappa\right)$, cf. Proposition 2.14(ii).

2.7. Inductive Freeness for Multiarrangements. As in the simple case, Theorem 2.12 motivates the notion of inductive freeness.

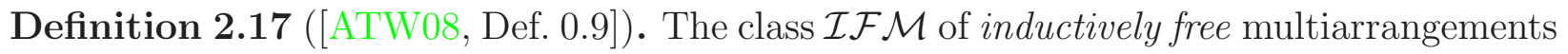
is the smallest class of multiarrangements subject to

(i) $\Phi_{\ell} \in \mathcal{I} \mathcal{F} \mathcal{M}$ for each $\ell \geq 0$;

(ii) for a multiarrangement $(\mathscr{A}, \mu)$, if there exists a hyperplane $H_{0} \in \mathscr{A}$ such that both $\left(\mathscr{A}^{\prime}, \mu^{\prime}\right)$ and $\left(\mathscr{A}^{\prime \prime}, \mu^{*}\right)$ belong to $\mathcal{I} \mathcal{F} \mathcal{M}$, and $\exp \left(\mathscr{A}^{\prime \prime}, \mu^{*}\right) \subseteq \exp \left(\mathscr{A}^{\prime}, \mu^{\prime}\right)$, then $(\mathscr{A}, \mu)$ also belongs to $\mathcal{I F} \mathcal{F}$.

Remark 2.18. As for simple arrangements, if $r(\mathscr{A}) \leq 2$, then $(\mathscr{A}, \mu)$ is inductively free, [Z89, Cor. 7].

Remark 2.19. In analogy to the simple case, cf. [OT92, §4.3, p. 119], [HR15, Rem. 2.9], it is possible to describe an inductively free multiarrangement $(\mathscr{A}, \mu)$ by means of a so called induction table. In this process we start with an inductively free multiarrangement (frequently $\Phi_{\ell}$ ) and add hyperplanes successively ensuring that part (ii) of Definition 2.17 is satisfied. We refer to this process as induction of hyperplanes. This procedure amounts to choosing an order on consecutive multiplicities $\mu_{i}$ of the simple arrangement $\mathscr{A}$ for $i=$ $1, \ldots, n=|\mu|$ (see Definition 2.5), so that $\left|\mu_{i}\right|=i, \mu_{n}=\mu$, and each restriction $\left(\mathscr{A}^{\prime \prime}, \mu_{i}^{*}\right)$ is inductively free. As in the simple case, in the associated induction table we record in the $i$-th row the information of the $i$-th step of this process, by listing $\exp \left(\mathscr{A}^{\prime}, \mu_{i}^{\prime}\right)=\exp \left(\mathscr{A}, \mu_{i-1}\right)$, the defining form $\alpha_{H_{i}}$ of $H_{i}$, as well as $\exp \left(\mathscr{A}^{\prime \prime}, \mu_{i}^{*}\right)$, For instance, see Tables 1, 3, and 4 below.

We record an easy consequence of Proposition 2.14(ii) in the context of inductive freeness which is going to be very useful in our proof of Theorem 1.9. 
Corollary 2.20. Suppose that both $\mathscr{A}$ and $\left(\mathscr{A}^{\prime \prime}, \kappa\right)$ are inductively free. Then $(\mathscr{A}, \delta)$ is inductively free.

Proof. The result follows readily from induction on $m_{0} \geq 1$, Proposition 2.14(ii) and a repeated application of the addition part of Theorem 2.12.

The following special case of Corollary 2.20 is immediate from [Z89, Cor. 7].

Corollary 2.21. Suppose that the rank 3 arrangement $\mathscr{A}$ is inductively free. Then $(\mathscr{A}, \delta)$ is inductively free.

The following is is a very useful tool for showing that a given multiarrangement is not inductively free by exhibiting a localization which fails to be inductively free.

Theorem 2.22 ([HRS17, Thm. 1.3]). The class $\mathcal{I F M}$ is closed under taking localizations.

We also require the fact that inductive freeness for multiarrangements behave well with the product construction.

Theorem 2.23 ([HRS17, Thm. 1.4]). A product of multiarrangements belongs to $\mathcal{I} \mathcal{F} \mathcal{M}$ if and only if each factor belongs to $\mathcal{I} \mathcal{F} \mathcal{M}$.

We close this section with an example which shows that the reverse implication of Corollary 1.5 fails in general.

Example 2.24. Consider the complex 4-arrangement $\mathscr{A}$ given by the defining polynomial $Q(\mathscr{A}):=x y z t(x-y)(x-z)(x-y+t)(x-z+t)$. Then one checks that $\mathscr{A}^{H_{t}}$ is inductively free, while both $\mathscr{A}$ and $\left(\mathscr{A}^{H_{t}}, \kappa\right)$ are not free.

\section{Filtrations of Free Multiplicities}

In the sequel, we denote by $\mathbb{1}$ the simple multiplicity on a given arrangement. Recall the partial order on the set of multiplicities on an arrangement from Definition 2.5.

Definition 3.1. Let $\mathscr{A}$ be a free arrangement. Suppose there is a free multiplicity $\mu>\mathbb{1}$ on $\mathscr{A}$ such that there is a sequence of free multiplicities $\mu_{i}$ on $\mathscr{A}$ satisfying $\mu_{i}<\mu_{i+1}$ and $\left|\mu_{i+1}\right|=\left|\mu_{i}\right|+1$, for $i=1, \ldots, n-1$, where $\mu_{1}:=\mathbb{1}$ and $\mu_{n}:=\mu$. Then we say that the sequence of multiarrangements $\left(\mathscr{A}, \mu_{i}\right)$ is a free filtration of $(\mathscr{A}, \mu)$ or simply that the sequence $\mu_{i}$ is a filtration of free multiplicities on $\mathscr{A}$.

Lemma 3.2. Let $\mathscr{A}$ be an inductively free arrangement. Suppose there is a free multiplicity $\mu>\mathbb{1}$ on $\mathscr{A}$ along with a free filtration $\mathbb{1}=: \mu_{1}<\ldots<\mu_{n}:=\mu$ of $(\mathscr{A}, \mu)$ as in Definition 3.1. If each restriction along the free filtration is inductively free, then so is $(\mathscr{A}, \mu)$.

Proof. It follows from Theorem 2.10 that the exponents of $\left(\mathscr{A}^{\prime}, \mu_{i}^{\prime}\right)=\left(\mathscr{A}, \mu_{i-1}\right)$ and of $\left(\mathscr{A}, \mu_{i}\right)$ differ in precisely one entry by 1 . So that, by Theorem 2.12 , the exponents of each restriction along the free filtration satisfy $\exp \left(\mathscr{A}^{\prime \prime}, \mu_{i}^{*}\right) \subseteq \exp \left(\mathscr{A}^{\prime}, \mu_{i}^{\prime}\right)$. Now, since $\mathscr{A}$ as well as each restriction $\left(\mathscr{A}^{\prime \prime}, \mu_{i}^{*}\right)$ is inductively free, a repeated application of the addition part of Theorem 2.12 gives the desired result. 
We record a special case of Lemma 3.2.

Corollary 3.3. Let $\mathscr{A}$ be an inductively free 3-arrangement. Suppose there is a free multiplicity $\mu \geq \mathbb{1}$ on $\mathscr{A}$ along with a free filtration as in Lemma 3.2. Then $(\mathscr{A}, \mu)$ is inductively free.

Proof. Since each restriction along the free filtration is of rank 2, it is already inductively free, by Remark 2.18. So the result is immediate from Corollary 3.3.

In our next result we present a mild condition on a free multiplicity $\mu$ of a free arrangement $\mathscr{A}$ which implies that every intermediate multiplicity $\mathbb{1}<\nu<\mu$ is also free.

Lemma 3.4. Let $\mathscr{A}$ be a free arrangement with exponents $1 \leq e_{2} \leq \ldots \leq e_{\ell}$. Assume that there is a free multiplicity $\mu>\mathbb{1}$ on $\mathscr{A}$ with $\exp (\mathscr{A}, \mu)=\left\{e, e_{2}, \ldots, e_{\ell}\right\}$, where $e=$ $1+|\mu|-|\mathscr{A}|$. Suppose that $|\mu|-|\mathscr{A}| \geq e_{\ell}$. Let $\nu$ be a multiplicity satisfying $\mathbb{1}<\nu<\mu$. Then $(\mathscr{A}, \nu)$ is free with $\exp (\mathscr{A}, \nu)=\left\{\tilde{e}, e_{2}, \ldots, e_{\ell}\right\}$, where $\tilde{e}=1+|\nu|-|\mathscr{A}|$.

Moreover, if $\theta_{1}, \ldots, \theta_{\ell}$ is a homogeneous basis of $D(\mathscr{A}, \mu)$ with $\operatorname{pdeg} \theta_{i}=e_{i}$ for $i=2, \ldots, \ell$ and $\operatorname{pdeg} \theta_{1}=e$, then

$$
\left(\prod_{H \in \mathscr{A}} \alpha_{H}^{\nu(H)-1}\right) \theta_{E}, \theta_{2}, \ldots, \theta_{\ell}
$$

is a basis of $D(\mathscr{A}, \nu)$.

Proof. Let $\theta_{1}, \ldots, \theta_{\ell}$ be a homogeneous basis of $D(\mathscr{A}, \mu)$ with $\operatorname{pdeg} \theta_{i}=e_{i}$ for $i=2, \ldots, \ell$ and pdeg $\theta_{1}=e=1+|\mu|-|\mathscr{A}|$. It is enough to show that $\theta_{E}, \theta_{2}, \ldots, \theta_{\ell}$ is a basis of $D(\mathscr{A})$. For, then the derivations given in (3.5) are linearly independent over $S$ and obviously also members of $D(\mathscr{A}, \nu)($ as $\nu \leq \mu)$ so that the sum over their polynomial degrees equals $|\nu|$, as $\sum_{i=2}^{\ell} e_{i}=|\mathscr{A}|-1$. We are going to check that the conditions of [OT92, Thm. 4.42] hold. For that we have to show that

$$
\theta_{i} \notin S \theta_{E}+S \theta_{2}+\cdots+S \theta_{i-1}
$$

holds for $i=2, \ldots, \ell$. Assume that (3.6) fails for some $i$. Then there are polynomials $p, p_{2}, \ldots, p_{i-1} \in S$ such that

$$
p \theta_{E}=\sum_{k=2}^{i-1} p_{k} \theta_{k}+\theta_{i} .
$$

Consequently, since $\theta_{2}, \ldots, \theta_{i}$ are linearly independent over $S, p \neq 0$. Now applying (3.7) to $\alpha_{H}$ for $H \in \mathscr{A}$, we get that $p \cdot \alpha_{H} \in \alpha_{H}^{\mu(H)} S$. Hence

$$
p \in\left(\prod_{H \in \mathscr{A}} \alpha_{H}^{\mu(H)-1}\right) S .
$$

Therefore, $\operatorname{deg} p \geq \sum_{H \in \mathscr{A}}(\mu(H)-1)=|\mu|-|\mathscr{A}|$. By (3.7), we have $1+\operatorname{deg} p=\operatorname{pdeg}\left(p \theta_{E}\right)=$ $\operatorname{pdeg} \theta_{i}$. Using the hypothesis $|\mu|-|\mathscr{A}| \geq e_{\ell}$, we get

$$
\operatorname{pdeg} \theta_{i} \geq 1+|\mu|-|\mathscr{A}|>|\mu|-|\mathscr{A}| \geq e_{\ell}=\operatorname{pdeg} \theta_{\ell} \geq \operatorname{pdeg} \theta_{i}
$$


which is absurd. Consequently, (3.6) holds for all $i=2, \ldots, \ell$ and so $\theta_{E}, \theta_{2}, \ldots, \theta_{\ell}$ is a basis of $D(\mathscr{A})$, as claimed, by loc. cit.

We record an important consequence of Lemma 3.4 and Theorem 2.12 which shows that the multisets of exponents of the restrictions along a free filtration, as in Lemma 3.4, are constant and do not depend on the multiplicities $\mu_{i}^{*}$.

Corollary 3.8. Let $\mathscr{A}$ and $(\mathscr{A}, \mu)$ be as in Lemma 3.4. Let $\mathbb{1}=: \mu_{1}<\ldots<\mu_{n}:=$ $\mu$ be a free filtration of $(\mathscr{A}, \mu)$ as in Definition 3.1. Then for each restriction, we have $\left(\mathscr{A}^{\prime \prime}, \mu_{i}^{*}\right)=\left(\mathscr{A}^{\prime \prime}, \kappa\right)$, where $\kappa$ is Ziegler's canonical multiplicity on $\mathscr{A}^{\prime \prime}$. In particular, each such restriction along the filtration is free with $\exp \left(\mathscr{A}^{\prime \prime}, \mu_{i}^{*}\right)=\exp \left(\mathscr{A}^{\prime \prime}, \kappa\right)=\left\{e_{2}, \ldots, e_{\ell}\right\}$, where $\exp \mathscr{A}=\left\{1, e_{2}, \ldots, e_{\ell}\right\}$.

Proof. Let $H_{0} \in \mathscr{A}$ and let $\mathbb{1} \leq \nu^{\prime}<\nu \leq \mu$ be free multiplicities as given by Lemma 3.4, where $\left(\mathscr{A}^{\prime}, \nu^{\prime}\right)$ is the deletion of $(\mathscr{A}, \nu)$ with respect to $H_{0}$. Let $\theta_{1}, \theta_{2}, \ldots, \theta_{\ell}$ be a free basis of $D\left(\mathscr{A}^{\prime}, \nu^{\prime}\right)$. It follows from Lemma 3.4 that $\alpha_{0} \theta_{1}, \theta_{2}, \ldots, \theta_{\ell}$ is a free basis of $D(\mathscr{A}, \nu)$, as given by (3.5), where ker $\alpha_{0}=H_{0}$. Owing to Theorem 2.11 , we see that $\bar{\theta}_{2}, \ldots, \bar{\theta}_{\ell}$ is a basis of $D\left(\mathscr{A}^{\prime \prime}, \nu^{*}\right)$.

Now let $\delta$ be the concentrated multiplicity on $\mathscr{A}$ given by $\delta\left(H_{0}\right)=2$. Then $\mathbb{1}<\delta \leq \mu$. Hence, according to Lemma $3.4,(\mathscr{A}, \delta)$ is free with $\alpha_{0} \theta_{E}, \theta_{2}, \ldots, \theta_{\ell}$ being a basis of $D(\mathscr{A}, \delta)$, by (3.5). Thanks to Proposition $2.14,\left(\mathscr{A}^{\prime \prime}, \delta^{*}\right)=\left(\mathscr{A}^{\prime \prime}, \kappa\right)$.

Since $\theta_{E}, \theta_{2}, \ldots, \theta_{\ell}$ is a basis of $D(\mathscr{A})$, it follows again from Theorem 2.11 applied to $(\mathscr{A}, \delta)$ and $\left(\mathscr{A}^{\prime}, \delta^{\prime}\right)=(\mathscr{A}, \mathbb{1})$, that $\bar{\theta}_{2}, \ldots, \bar{\theta}_{\ell}$ is also a basis of $D\left(\mathscr{A}^{\prime \prime}, \kappa\right)$. Hence, thanks to $(2.7)$, $\nu^{*}=\kappa$.

Next we apply Lemma 3.4 in the context of Ziegler's canonical multiplicity $\kappa$.

Corollary 3.9. Let $\mathscr{A}$ be a free arrangement with exponents $1=e_{1} \leq e_{2} \leq \cdots \leq e_{\ell-1}<e_{\ell}$ and let $H \in \mathscr{A}$ such that $\mathscr{A}^{H}$ is free with $\exp \left(\mathscr{A}^{H}\right)=\left\{1, e_{2}, \ldots, e_{\ell-1}\right\}$. Then $\left(\mathscr{A}^{H}, \mu\right)$ is free for every multiplicity $\mathbb{1}<\mu<\kappa$ with $\exp \left(\mathscr{A}^{H}, \mu\right)=\left\{1+|\mu|-\left|\mathscr{A}^{H}\right|, e_{2}, \ldots, e_{\ell-1}\right\}$.

Proof. Since $\mathscr{A}^{H}$ is free with $\exp \left(\mathscr{A}^{H}\right)=\left\{1, e_{2}, \ldots, e_{\ell-1}\right\}$, we have $\left|\mathscr{A}^{H}\right|=1+e_{2}+\ldots+e_{\ell-1}$. Also note that $|\kappa|=|\mathscr{A}|-1$. Therefore,

$$
1+|\kappa|-\left|\mathscr{A}^{H}\right|=|\mathscr{A}|-\left(1+e_{2}+\ldots+e_{\ell-1}\right)=e_{\ell} .
$$

Consequently, $\exp \left(\mathscr{A}^{H}, \kappa\right)=\left\{e_{\ell}, e_{2}, \ldots, e_{\ell-1}\right\}=\left\{1+|\kappa|-\left|\mathscr{A}^{H}\right|, e_{2}, \ldots, e_{\ell-1}\right\}$, by Theorem 1.2. Thus $|\kappa|-\left|\mathscr{A}^{H}\right|=e_{\ell}-1 \geq e_{\ell-1}$, by hypothesis. So the result follows from Lemma 3.4 applied to $\mathscr{A}^{H}$ and $\kappa$.

Corollary 3.9 yields that under rather weak assumptions, every multiplicity between $\mathbb{1}$ and the Ziegler multiplicity $\kappa$ is free. This yields an abundance of free filtrations from $\mathbb{1}$ to $\kappa$ on $\mathscr{A}^{H}$. We demonstrate this in the context of reflection arrangements.

Proposition 3.10. Let $W$ be of type $A_{\ell}, B_{\ell}, G(r, 1, \ell), F_{4}, H_{4}, G_{31}, G_{32} E_{6}, E_{7}$, or $E_{8}$. Let $\mathscr{A}=\mathscr{A}(W)$. Then for any $H \in \mathscr{A}$ and every multiplicity $\mathbb{1} \leq \mu \leq \kappa$, the multiarrangement $\left(\mathscr{A}^{H}, \mu\right)$ is free with $\exp \left(\mathscr{A}^{H}, \mu\right)=\left\{1+|\mu|-\left|\mathscr{A}^{H}\right|, e_{2}, \ldots, e_{\ell-1}\right\}$. 
Proof. It readily follows from [OT92, Props. 6.73, 6.77] and the tables in [OT92, App. C] that in the given cases any restriction $\mathscr{A}^{H}$ satisfies the condition of Corollary 3.9.

Armed with Corollaries 3.8 and 3.9, we can now prove our first main result of our classification.

Proposition 3.11. Let $\mathscr{A}=\mathscr{A}(W)$ be the reflection arrangement of the full monomial group $W=G(r, 1, \ell)$ for $r, \ell \geq 2$. Then both $\left(\mathscr{A}^{\prime \prime}, \kappa\right)$ and $(\mathscr{A}, \delta)$ are inductively free.

Proof. Owing to Theorem 2.3 and Corollary 2.20, it is enough to show that $\left(\mathscr{A}^{\prime \prime}, \kappa\right)$ is inductively free. We prove the latter by induction on $\ell$. Clearly, for $\ell \leq 3$, the statement holds. Thus suppose that $\ell \geq 4$ and that the result is true for smaller ranks.

Note that irrespective of the choice of hyperplane in $\mathscr{A}$, we have

$$
\mathscr{A}^{\prime \prime} \cong \mathscr{A}(G(r, 1, \ell-1)) \text {, }
$$

by [OS82, Prop. 2.11] (cf. [OT92, Prop. 6.82]). Owing to [OS82, Prop. 2.13] (cf. [OT92, Prop. 6.77]), we have

$$
\exp \mathscr{A}=\{1, r+1, \ldots,(\ell-1) r+1\}
$$

Thanks to Proposition 3.10, each multiplicity $\mu$ on $\mathscr{A}^{\prime \prime}$ with $\mathbb{1} \leq \mu \leq \kappa$ is free. So we pick an arbitrary free filtration of $\mathscr{A}^{\prime \prime}$ from $\mathbb{1}$ to $\kappa$. Since $|\kappa|-\left|\mathscr{A}^{\prime \prime}\right|=(\ell-1) r>(\ell-2) r+1$, which is the highest exponent of $\mathscr{A}^{\prime \prime}$, by (3.12) and (3.13), the hypotheses of Lemma 3.4 are satisfied for $\mathscr{B}:=\mathscr{A}^{\prime \prime}$. Consequently, Corollary 3.8 implies that for each multiplicity $\mu$ along our free chain we have $\left(\mathscr{B}^{\prime \prime}, \mu^{*}\right)=\left(\mathscr{B}^{\prime \prime}, \kappa_{1}\right)$, where $\kappa_{1}$ denotes the Ziegler multiplicity on $\mathscr{B}^{\prime \prime}$. Since $\mathscr{B}^{\prime \prime} \cong \mathscr{A}(G(r, 1, \ell-2))$, by (3.12), it follows from our induction hypothesis that $\left(\mathscr{B}^{\prime \prime}, \kappa_{1}\right)$ is inductively free. Consequently, since the simple arrangement $\mathscr{A}^{\prime \prime}$ is inductively free, thanks to Theorem 2.3, it follows that $\left(\mathscr{A}^{\prime \prime}, \kappa\right)$ is inductively free, by a repeated application of the addition part of Theorem 2.12 .

Moreover, Corollaries 3.3 and 3.9, imply the following consequence of Theorem 2.4.

Corollary 3.14. Let $W$ be of type $F_{4}, H_{4}, G_{31}$ or $G_{32}$. Then $\left(\mathscr{A}(W)^{\prime \prime}, \kappa\right)$ is inductively free.

Proof. By Proposition 3.10, in each of the given cases the condition in Corollary 3.9 on the exponents of $\mathscr{A}(W)^{\prime \prime}$ is satisfied, so that every chain of multiplicities between $\mathbb{1}$ and $\kappa$ on $\mathscr{A}(W)^{\prime \prime}$ is free. By Theorem 2.4, $\mathscr{A}(W)^{\prime \prime}$ is inductively free in each instance. Therefore, it follows from Corollary 3.3 that also $\left(\mathscr{A}(W)^{\prime \prime}, \kappa\right)$ is inductively free.

\section{Proofs of Theorems 1.4 And 1.9}

Throughout, $W$ denotes a complex reflection group. Owing to [HR15, Prop. 2.10] and Theorem 2.23, we may assume that $W$ is irreducible. Fix $H_{0} \in \mathscr{A}$ and let $\mathscr{A}^{\prime \prime}$ be the restriction of $\mathscr{A}$ with respect to $H_{0}$. Moreover, fix $m_{0} \in \mathbb{Z}_{\geq 1}$ and let $\delta=\delta_{H_{0}, m_{0}}$ as in Definition 1.6. We prove Theorems 1.4 and 1.9 more less simultaneously making repeated use of Corollary 2.20. 
4.1. Groups of low rank. It is immediate from [Z89, Cor. 7] that the reverse implication in Theorem 1.4 holds provided $W$ has rank at most 3. Likewise Theorem 1.9 holds for $W$ of rank at most 2 .

4.2. Coxeter Groups. Let $W$ be an irreducible Coxeter group. By [BC12], every Coxeter arrangement is inductively free. So once we have shown that $\left(\mathscr{A}(W)^{\prime \prime}, \kappa\right)$ is inductively free, so is $(\mathscr{A}(W), \delta)$, thanks to Corollary 2.20.

Lemma 4.1. Let $\mathscr{A}=\mathscr{A}\left(A_{\ell-1}\right)$ be the Coxeter arrangement of type $A_{\ell-1}$. Then both $\left(\mathscr{A}^{\prime \prime}, \kappa\right)$ and $(\mathscr{A}, \delta)$ are inductively free.

Proof. For $\ell \leq 4$, the result follows from $\S 4.1$ and Corollary 2.21. So suppose that $\ell \geq 5$. Since the underlying Coxeter group is transitive on $\mathscr{A}$, there is no harm in fixing $H_{0}=$ $\operatorname{ker}\left(x_{1}-x_{2}\right)$. Thanks to [OT92, Prop. 6.73], $\mathscr{A}^{\prime \prime} \cong \mathscr{A}\left(A_{\ell-2}\right)$. One readily checks that Ziegler's multiarrangement $\left(\mathscr{A}\left(A_{\ell-2}\right), \kappa\right)$ has defining polynomial

$$
Q\left(\mathscr{A}\left(A_{\ell-2}\right), \kappa\right)=\prod_{2 \leq j \leq \ell-2}\left(x_{1}-x_{j}\right)^{2} \prod_{2 \leq i<j \leq \ell-2}\left(x_{i}-x_{j}\right) .
$$

It follows from [CR16, Cor. 1.2] that $\left(\mathscr{A}\left(A_{\ell-2}\right), \kappa\right)$ is inductively free. Since $\mathscr{A}$ is inductively free, it follows from Corollary 2.20 that $(\mathscr{A}, \delta)$ is inductively free.

The following is the special case $r=2$ in Proposition 3.11.

Lemma 4.2. Let $\mathscr{A}=\mathscr{A}\left(B_{\ell}\right)$ be the Coxeter arrangement of type $B_{\ell}$. Then both $\left(\mathscr{A}^{\prime \prime}, \kappa\right)$ and $(\mathscr{A}, \delta)$ are inductively free.

Lemma 4.3. Let $\mathscr{A}=\mathscr{A}\left(D_{\ell}\right)$ be the Coxeter arrangement of type $D_{\ell}$ for $\ell \geq 3$. Then both $\left(\mathscr{A}^{\prime \prime}, \kappa\right)$ and $(\mathscr{A}, \delta)$ are inductively free.

Proof. We first prove that $\left(\mathscr{A}^{\prime \prime}, \kappa\right)$ is inductively free. We argue by induction on $\ell \geq 3$. For $\ell=3$, we have $\mathscr{A}\left(D_{3}\right)=\mathscr{A}\left(A_{3}\right)$ and so then the result follows from Lemma 4.1.

Now let $W=W\left(D_{\ell}\right)$ for $\ell \geq 4$ and suppose that the result holds for all instances of smaller rank. Since $W$ is transitive on $\mathscr{A}$, we may fix $H_{0}=\operatorname{ker}\left(x_{1}-x_{2}\right)$ without loss. It follows from [OT92, Prop. 6.85] that $\mathscr{A}^{\prime \prime}=\mathscr{A}_{\ell-1}^{1}(2)$.

One readily checks that Ziegler's multiarrangement $\left(\mathscr{A}\left(D_{\ell}\right)^{\prime \prime}, \kappa\right)$ has defining polynomial

$$
Q\left(\mathscr{A}\left(D_{\ell}\right)^{\prime \prime}, \kappa\right)=Q\left(\mathscr{A}_{\ell-1}^{1}(2), \kappa\right)=x_{1} \prod_{2 \leq j \leq \ell-1}\left(x_{1}^{2}-x_{j}^{2}\right)^{2} \prod_{3 \leq i<j \leq \ell-1}\left(x_{i}^{2}-x_{j}^{2}\right) .
$$

According to Theorem 1.2, $\left(\mathscr{A}\left(D_{\ell}\right)^{\prime \prime}, \kappa\right)$ is free with

$$
\exp \left(\mathscr{A}\left(D_{\ell}\right)^{\prime \prime}, \kappa\right)=\exp \left(\mathscr{A}_{\ell-1}^{1}(2), \kappa\right)=\{3,5, \ldots, 2(\ell-3)+1, \ell-1\} .
$$

By our induction hypothesis and Theorem 2.23, the sub-multiarrangement $\left(\mathscr{A}\left(D_{\ell-1}\right)^{\prime \prime}, \kappa\right) \times$ $\Phi_{1}$ of $\left(\mathscr{A}\left(D_{\ell}\right)^{\prime \prime}, \kappa\right)$ is inductively free. By (4.4), its set of exponents is

$$
\exp \left(\left(\mathscr{A}\left(D_{\ell-1}\right)^{\prime \prime}, \kappa\right) \times \Phi_{1}\right)=\exp \left(\left(\mathscr{A}_{\ell-2}^{1}(2), \kappa\right) \times \Phi_{1}\right)=\{3,5, \ldots, 2(\ell-4)+1, \ell-2,0\} .
$$

This forms the start of our induction table. 
We first add and restrict to the hyperplanes $\operatorname{ker}\left(x_{1}-x_{\ell-1}\right)$ and $\operatorname{ker}\left(x_{1}+x_{\ell-1}\right)$ twice. Here we use Proposition 2.13 in order to determine the Euler multiplicity on the restrictions. We consider the special case when $\operatorname{ker}\left(x_{1}+x_{\ell-1}\right)$ is added and restricted to for the second time. Then in the notation of Proposition 2.13, when considering the localization $\mathscr{A}_{X}=$ $\left\{\operatorname{ker}\left(x_{1}+x_{\ell-1}\right), \operatorname{ker}\left(x_{1}-x_{\ell-1}\right), \operatorname{ker} x_{1}\right\}$, we have $\left|\mathscr{A}_{X}\right|=3,\left|\mu_{X}\right|=5$ and so Proposition 2.13(2) applies.

Note that each of the restrictions is isomorphic to $\left(\mathscr{A}_{\ell-2}^{1}(2), \kappa\right)$ which is inductively free, by our inductive hypothesis. Consequently, since in each case both $\left(\mathscr{A}^{\prime}, \mu^{\prime}\right)$ and $\left(\mathscr{A}^{\prime \prime}, \mu^{*}\right)$ are inductively free and $\exp \left(\mathscr{A}^{\prime \prime}, \mu^{*}\right) \subseteq \exp \left(\mathscr{A}^{\prime}, \mu^{\prime}\right)$, it follows that also $(\mathscr{A}, \mu)$ is inductively free.

\begin{tabular}{lll}
\hline $\exp \left(\mathscr{A}^{\prime}, \mu^{\prime}\right)$ & $\alpha_{H}$ & $\exp \left(\mathscr{A}^{\prime \prime}, \mu^{*}\right)$ \\
\hline \hline $\exp \left(\left(\mathscr{A}_{\ell-2}^{1}(2), \kappa\right) \times \Phi_{1}\right)$ & $x_{1}-x_{\ell-1}$ & $\exp \left(\mathscr{A}_{\ell-2}^{1}(2), \kappa\right)$ \\
$=\{3,5, \ldots, 2(\ell-4)+1, \ell-2,0\}$ & & \\
$\{3,5, \ldots, 2(\ell-4)+1, \ell-2,1\}$ & $x_{1}+x_{\ell-1}$ & $\exp \left(\mathscr{A}_{\ell-2}^{1}(2), \kappa\right)$ \\
$\{3,5, \ldots, 2(\ell-4)+1, \ell-2,2\}$ & $x_{1}-x_{\ell-1}$ & $\exp \left(\mathscr{A}_{\ell-2}^{1}(2), \kappa\right)$ \\
$\{3,5, \ldots, 2(\ell-4)+1, \ell-2,3\}$ & $x_{1}+x_{\ell-1}$ & $\exp \left(\mathscr{A}_{\ell-2}^{1}(2), \kappa\right)$ \\
$\{3,5, \ldots, 2(\ell-4)+1, \ell-2,4\}$ & $x_{2}-x_{\ell-1}$ & $\exp \left(\mathscr{A}_{\ell-2}^{1}(2), \kappa\right)$ \\
$\vdots$ & $\vdots$ & $\vdots$ \\
$\{3,5, \ldots, 2(\ell-4)+1,2(\ell-3)+1, \ell-2\}$ & $x_{\ell-2}+x_{\ell-1}$ & $\exp \left(\mathscr{A}_{\ell-2}^{1}(2), \kappa\right)$ \\
$\exp \left(\mathscr{A}\left(D_{\ell}\right)^{\prime \prime}, \kappa\right)=\{3,5, \ldots, 2(\ell-3)+1, \ell-1\}$ & & \\
\hline
\end{tabular}

TABle 1. Induction Table for $\left(\mathscr{A}\left(D_{\ell}\right)^{\prime \prime}, \kappa\right)$.

It follows from Table 1 that $\left(\mathscr{A}\left(D_{\ell}\right)^{\prime \prime}, \kappa\right)$ is inductively free. Since $\mathscr{A}$ itself is inductively free, so is $(\mathscr{A}, \delta)$, according to Corollary 2.20 .

Lemma 4.5. Let $W$ be an irreducible Coxeter group of exceptional type of rank at least 3 and let $\mathscr{A}=\mathscr{A}(W)$ be the Coxeter arrangement of $W$. Then both $\left(\mathscr{A}^{\prime \prime}, \kappa\right)$ and $(\mathscr{A}, \delta)$ are inductively free.

Proof. For $W$ of type $H_{3}$ the result follows from Corollary 2.21.

For the remaining instances, we first prove that $\left(\mathscr{A}^{\prime \prime}, \kappa\right)$ is inductively free. For $W$ of type $F_{4}$ and $H_{4}$, this follows from Corollary 3.14.

Since $W\left(E_{6}\right)$ and $W\left(E_{7}\right)$ are parabolic subgroups of $W\left(E_{8}\right)$, we obtain the result for the former from the latter, thanks to [OT92, Cor. 6.28], Lemma 2.16(ii), and Theorem 2.22.

The case for $W=W\left(E_{8}\right)$ is considerably more complicated. Since $W$ is transitive on $\mathscr{A}$, we may take $H_{0}=\operatorname{ker} x$. It follows from Proposition 3.10 that $\left(\mathscr{A}^{\prime \prime}, \nu\right)$ is free for every multiplicity $\nu$ with $\mathbb{1} \leq \nu \leq \kappa$. So we pick a filtration $\mathbb{1}=\nu_{1}<\nu_{2} \ldots<\nu_{28}<\nu_{29}=$ $\kappa$ of free multiplicities. Since $\mathscr{B}:=\mathscr{A}^{\prime \prime}$ is inductively free, by [BC12], it follows from 
Lemma 3.2 that $\left(\mathscr{A}^{\prime \prime}, \kappa\right)$ is inductively free, once we have established that the corresponding multirestrictions $\left(\mathscr{B}^{\prime \prime}, \nu_{i}^{*}\right)$ are inductively free. Moreover, each multiplicity $\nu_{i}^{*}$ is just Ziegler's canonical multirestriction $\kappa_{1}$ stemming from the simple arrangement $\mathscr{B}$, by Corollary 3.8. Let $\mathscr{C}:=\mathscr{B}^{\prime \prime}$. Since $\mathscr{C}$ is inductively free, by [BC12], we can build an induction table by starting with the simple arrangement $\mathscr{C}$ and increasing the multiplicities all the way to $\left(\mathscr{C}, \kappa_{1}\right)$. This is carried out in Table 2 , where we indicate how to increase the multiplicities on the hyperplanes of the inductively free arrangement $\mathscr{C}$ such that each resulting multiplicity is again a free multiplicity for $\mathscr{C}$. Note that in the first 16 steps of the table the Euler multiplicity $\mu^{*}$ of the multirestriction is just Ziegler's canonical restriction on the simple arrangement $\mathscr{C}^{\prime \prime}$. The remaining two Euler multiplicities are small extensions of Ziegler's canonical multiplicity. In particular, here the order in which the hyperplanes are added plays a crucial role. There are precisely 3 different rank 5 multiarrangements that occur as restrictions in Table 2. We have checked that each of them is again inductively free, as required. However, we omit to include this data. We also do not give linear forms for $\alpha_{H}$ in the table, but give the number of the induction step at the beginning of each row.

\begin{tabular}{rlll}
\hline Step & $\exp \left(\mathscr{A}^{\prime}, \mu^{\prime}\right)$ & $\alpha_{H}$ & $\exp \left(\mathscr{A}^{\prime \prime}, \mu^{*}\right)$ \\
\hline \hline 0 & $\exp (\mathscr{C})=\{1,7,11,13,14,17\}$ & $\ldots$ & $\{7,11,13,14,17\}$ \\
& $\vdots$ & $\vdots$ & $\vdots$ \\
15 & $\{7,11,13,14,16,17\}$ & $\ldots$ & $\{7,11,13,14,17\}$ \\
16 & $\{7,11,13,14,17,17\}$ & $\ldots$ & $\{7,11,13,17,17\}$ \\
& $\vdots$ & $\vdots$ & $\vdots$ \\
20 & $\{7,11,13,17,17,18\}$ & $\ldots$ & $\{7,11,13,17,17\}$ \\
21 & $\{7,11,13,17,17,19\}$ & $\ldots$ & $\{7,11,13,17,19\}$ \\
& $\vdots$ & $\vdots$ & $\vdots$ \\
26 & $\{7,11,13,17,19,22\}$ & $\ldots$ & $\{7,11,13,17,19\}$ \\
& $\exp \left(\mathscr{C}, \kappa_{1}\right)=\{7,11,13,17,19,23\}$ & & \\
\hline
\end{tabular}

TABLE 2. Induction Table for $\left(\mathscr{C}, \kappa_{1}\right)$.

We mention that the only types of restrictions which do occur throughout this induction for $\left(\mathscr{A}^{\prime \prime}, \kappa\right)$ are $\left(E_{8}, A_{2}\right)$ in rank $6,\left(E_{8}, A_{3}\right)$ in $\operatorname{rank} 5,\left(E_{8}, A_{4}\right)$ and $\left(E_{8}, D_{4}\right)$ in rank 4 and $\left(E_{8}, A_{1} \times D_{4}\right),\left(E_{8}, D_{5}\right)$ and $\left(E_{8}, A_{5}\right)$ in rank 3 , where we use the notation to indicate restrictions following [OS82, §3, App.] (cf. [OT92, §6.4, App. C]).

Finally, since $\left(\mathscr{A}^{\prime \prime}, \kappa\right)$ is inductively free in each instance, it follows again from Corollary 2.20 that also $(\mathscr{A}, \delta)$ is inductively free.

4.3. The Monomial Groups $G(r, 1, \ell)$. For $W=G(r, 1, \ell)$ for $r, \ell \geq 2$, the result follows from Proposition 3.11. 
4.4. Non-real inductively free reflection arrangements of exceptional type. Let $W$ be irreducible of rank at least 3 of exceptional type so that $\mathscr{A}=\mathscr{A}(W)$ is inductively free. It then follows from Theorem 2.3 that $W$ equals $G_{25}, G_{26}$, or $G_{32}$.

Lemma 4.6. Let $\mathscr{A}=\mathscr{A}(W)$ be the reflection arrangement where $W$ is $G_{25}, G_{26}$, or $G_{32}$. Then both $\left(\mathscr{A}^{\prime \prime}, \kappa\right)$ and $(\mathscr{A}, \delta)$ are inductively free.

Proof. Since $G_{25}$ and $G_{26}$ are both of rank 3, it follows from Corollary 2.21 that then $(\mathscr{A}(W), \delta)$ is inductively free. Since $\mathscr{A}^{\prime \prime}$ is of rank 2 , also $\left(\mathscr{A}^{\prime \prime}, \kappa\right)$ is inductively free.

For $W=G_{32}$ it follows from Corollary 3.14 that $\left(\mathscr{A}^{\prime \prime}, \kappa\right)$ is inductively free. Owing to Corollary 2.20 , also $(\mathscr{A}, \delta)$ is inductively free.

4.5. Non-inductively free reflection arrangements. Suppose that $W$ is irreducible so that $\mathscr{A}=\mathscr{A}(W)$ is not inductively free. Then, according to Theorem 2.3, either $W=$ $G(r, r, \ell)$, for $r, \ell \geq 3$ or $W$ is of exceptional type $G_{24}, G_{27}, G_{29}, G_{31}, G_{33}$, or $G_{34}$.

The following is [HRS17, Prop. 4.1], its proof consists of an application of Theorem 2.22.

Lemma 4.7. Let $\mathscr{A}=\mathscr{A}(W)$ be the reflection arrangement of the monomial group $W=$ $G(r, r, \ell)$ for $r \geq 3$ and $\ell \geq 5$. Then both $\left(\mathscr{A}^{\prime \prime}, \kappa\right)$ and $(\mathscr{A}, \delta)$ are not inductively free.

Lemma 4.8. Let $W$ be $G(r, r, 3), G(r, r, 4)$, for $r \geq 3$ or of exceptional type $G_{24}, G_{27}, G_{29}$, $G_{31}, G_{33}$, or $G_{34}$. Then $(\mathscr{A}(W), \delta)$ is not inductively free.

Proof. Let $\mathscr{A}=\mathscr{A}(W)$ be the reflection arrangement for $W$ as in the statement. We argue by induction on $m_{0} \geq 1$. For $m_{0}=1$, the result follows from Theorem 2.3. So suppose that $m_{0}>1$ and that the claim is proved for smaller values for $m_{0}$. Pick $H \in \mathscr{A}$ and let $\left(\mathscr{A}, \mathscr{A}^{\prime}, \mathscr{A}^{\prime \prime}\right)$ be the triple of $\mathscr{A}$ with respect to $H$. If $H=H_{0}$, then $\left(\mathscr{A}^{\prime}, \delta^{\prime}\right)=\left(\mathscr{A}, \delta_{H_{0}, m_{0}-1}\right)$ which is not inductively free by inductive hypothesis.

Now suppose that $H \neq H_{0}$. In each case apart from $G_{31}$ it follows from [HR15, Cor. 1.3, Cor. 2.4] that $\mathscr{A}^{\prime}=\mathscr{A} \backslash\{H\}$ is not free for any choice of hyperplane. Therefore, in each of these instances, since $\mathscr{A}^{\prime}$ is not free, neither is $\left(\mathscr{A}^{\prime}, \delta^{\prime}\right)$, by Theorem 1.7. In particular, $\left(\mathscr{A}^{\prime}, \delta^{\prime}\right)$ is not inductively free. Thus $\left(\mathscr{A}^{\prime}, \delta^{\prime}\right)$ is not inductively free for any choice of $H$ and therefore, neither is $(\mathscr{A}, \delta)$.

For $W$ of exceptional type $G_{31}$, we argue as follows. Suppose that $(\mathscr{A}, \delta)$ is inductively free. Let $\mathscr{A}^{\prime}=\mathscr{A} \backslash\{H\}$ and $\left(\mathscr{A}^{\prime}, \delta^{\prime}\right)=\left(\mathscr{A}^{\prime},\left.\delta\right|_{\mathscr{A}}\right)$. If $\left(\mathscr{A}^{\prime}, \delta^{\prime}\right)$ is free, then so is $\mathscr{A}^{\prime}$, again thanks to Theorem 1.7. Continuing in this fashion removing further hyperplanes from the multiarrangement, we obtain a free chain of consecutive subarrangements of the simple arrangement $\mathscr{A}\left(G_{31}\right)$ ending at the empty 4 -arrangement. But this contradicts the fact that there is no such free chain of $\mathscr{A}\left(G_{31}\right)$, according to the proof of [HR15, Lem. 3.5].

Lemma 4.9. Let $W$ be $G(r, r, 3), G(r, r, 4)$, for $r \geq 3$ or $W$ is exceptional of type $G_{29}$ or $G_{31}$. Then $\left(\mathscr{A}(W)^{\prime \prime}, \kappa\right)$ is inductively free.

Proof. For $W=G(r, r, 3)$ the result is clear, thanks to Remark 2.18, and for $W=G_{31}$ the result follows from Corollary 3.14. 
If $W=G(r, r, 4)$ or $W$ is of type $G_{29}$, then $\mathscr{A}(W)^{\prime \prime}$ is inductively free, according to Theorem 2.4 ( $W$ is transitive on $\mathscr{A}(W)$ in each of these cases). Consequently, we may initialize an induction table in these instances with the simple inductively free arrangement $\mathscr{A}(W)^{\prime \prime}$ and add hyperplanes to reach the Ziegler multiplicity $\kappa$.

Let $W=G(r, r, 4)$. Then, by [OT92, Prop. 6.82], for any choice $H_{0} \in \mathscr{A}$, we have $\mathscr{A}(W)^{\prime \prime} \cong$ $\mathscr{A}_{3}^{1}(r)$ which is inductively free, according to Theorem 2.4(ii). Fix $H_{0}=\operatorname{ker}\left(x_{1}-x_{4}\right)$. Thanks to [OT92, Cor. 6.86], we have

$$
\exp \mathscr{A}(G(r, r, 4))=\{1, r+1,2 r+1,3(r-1)\}
$$

and therefore, by Theorem $1.2,\left(\mathscr{A}(G(r, r, 4))^{\prime \prime}, \kappa\right)$ is free with

$$
\exp \left(\mathscr{A}(G(r, r, 4))^{\prime \prime}, \kappa\right)=\{r+1,2 r+1,3(r-1)\} .
$$

So in particular, $|\kappa|=6 r-1$. Moreover, by [OT92, Prop. 6.85], we have

$$
\exp \mathscr{A}(G(r, r, 4))^{\prime \prime}=\exp \mathscr{A}_{3}^{1}(r)=\{1, r+1,2 r-1\} .
$$

The defining polynomial for $\left(\mathscr{A}(G(r, r, 4))^{\prime \prime}, \kappa\right)$ is given by

$$
Q\left(\mathscr{A}(G(r, r, 4))^{\prime \prime}, \kappa\right)=Q\left(\mathscr{A}_{3}^{1}(r), \kappa\right)=x_{1}^{r-1}\left(x_{1}^{r}-x_{2}^{r}\right)^{2}\left(x_{1}^{r}-x_{3}^{r}\right)^{2}\left(x_{2}^{r}-x_{3}^{r}\right) .
$$

Table 3 is the induction table for $\left(\mathscr{A}(G(r, r, 4))^{\prime \prime}, \kappa\right)$. Note that since $\mathscr{A}(G(r, r, 4))^{\prime \prime}$ is of rank 3 , each of the restrictions in Table 3 is of rank 2 and so is inductively free. Let $\zeta$ be a primitive $r^{\text {th }}$ root of unity.

There are two different types of steps in this induction table. The first one consists of adding a hyperplane $\operatorname{ker}\left(x_{1}-\zeta^{i} x_{2}\right)$ for some $i$ or $\operatorname{ker}\left(x_{1}-\zeta^{j} x_{3}\right)$ for some $j$ and the second one consists of adding $\operatorname{ker} x_{1}$.

In the first step, our defining polynomial is of the form

$$
Q(\mathscr{A}, \mu)=x_{1}\left(x_{2}^{r}-x_{3}^{r}\right) \prod_{0 \leq i<r}\left(x_{1}-\zeta^{i} x_{2}\right)^{k_{i}}\left(x_{1}-\zeta^{i} x_{3}\right)^{l_{i}}
$$

with $k_{i}, l_{i} \in\{1,2\}$ for all $i$.

We claim that $(\mathscr{A}, \mu)$ is free with $\exp (\mathscr{A}, \mu)=\left\{1+\sum_{0 \leq i<r}\left(k_{i}+l_{i}-2\right), r+1,2 r-1\right\}$. This is true if $k_{i}=l_{i}=1$ for all $i$, since then $(\mathscr{A}, \mu)$ is the simple arrangement $\mathscr{A}_{3}^{1}(r)$. For each hyperplane in this step the argument is essentially the same, so we only present it for $H=\operatorname{ker}\left(x_{1}-\zeta^{i} x_{2}\right)$. Fix $i$ and let $k_{i}=1$. The defining polynomial of the restriction is

$$
Q\left(\mathscr{A}^{H}, \mu^{*}\right)=x_{1}^{r} \prod_{0 \leq j<r}\left(x_{1}-\zeta^{j} x_{3}\right)^{2} .
$$

The Euler multiplicities can be computed using Proposition 2.13(2) and (3). A basis of $D\left(\mathscr{A}^{H}, \mu^{*}\right)$ is given by

$$
\begin{aligned}
& \theta_{1}=r x_{1}^{r+1} D_{1}+\left((r+1) x_{1}^{r} x_{3}-x_{3}^{r+1}\right) D_{3}, \\
& \theta_{2}=x_{1}^{r-1}\left(r x_{1} x_{3}^{r-1} D_{1}+\left(x_{1}^{r}+(r-1) x_{3}^{r}\right)\right) D_{3} .
\end{aligned}
$$

Therefore, we have $\exp \left(\mathscr{A}^{H}, \mu^{*}\right)=\{r+1,2 r-1\}$. The Addition Deletion Theorem 2.12 then yields that $(\mathscr{A}, \mu)$ is free with the desired exponents. 
In the second step we consider defining polynomials of the form

$$
Q(\mathscr{A}, \mu)=x_{1}^{i}\left(x_{1}^{r}-x_{2}^{r}\right)^{2}\left(x_{1}^{r}-x_{3}^{r}\right)^{2}\left(x_{2}^{r}-x_{3}^{r}\right),
$$

for $1 \leq i \leq r-2$.

We claim that $(\mathscr{A}, \mu)$ is free with $\exp (\mathscr{A}, \mu)=\{r+1,2 r+1,2 r-1+i\}$. We know from the first step that this is true for $i=1$. Let $H=\operatorname{ker} x_{1}$. The restriction is given by

$$
Q\left(\mathscr{A}^{H}, \mu^{*}\right)=x_{2}^{r+1} x_{3}^{r+1}\left(x_{2}^{r}-x_{3}^{r}\right) .
$$

Note that $\left(\mathscr{A}^{H}, \mu^{*}\right)$ coincides with $\left(\mathscr{A}(G(r, 1,3))^{\operatorname{ker} x_{1}}, \kappa\right)$. Therefore, thanks to Theorem 1.2, $\left(\mathscr{A}^{H}, \mu^{*}\right)$ is free and $\exp \left(\mathscr{A}^{H}, \mu^{*}\right)=\{r+1,2 r+1\}$. Since the hypotheses of Theorem 2.12 are fulfilled, we get freeness of $(\mathscr{A}, \mu)$ along with the desired exponents for each $i$.

This completes the argument for $W=G(r, r, 4)$. We record the details of the induction in Table 3.

\begin{tabular}{lll}
\hline $\exp \left(\mathscr{A}^{\prime}, \mu^{\prime}\right)$ & $\alpha_{H}$ & $\exp \left(\mathscr{A}^{\prime \prime}, \mu^{*}\right)$ \\
\hline \hline $\exp \mathscr{A}_{3}^{1}(r)=\{1, r+1,2 r-1\}$ & $x_{1}-\zeta x_{2}$ & $\{r+1,2 r-1\}$ \\
$\{2, r+1,2 r-1\}$ & $x_{1}-\zeta^{2} x_{2}$ & $\{r+1,2 r-1\}$ \\
$\vdots$ & $\vdots$ & $\vdots$ \\
$\{r, r+1,2 r-1\}$ & $x_{1}-x_{2}$ & $\{r+1,2 r-1\}$ \\
$\{r+1, r+1,2 r-1\}$ & $x_{1}-\zeta x_{3}$ & $\{r+1,2 r-1\}$ \\
$\vdots$ & $\vdots$ & $\vdots$ \\
$\{r+1,2 r, 2 r-1\}$ & $x_{1}-x_{3}$ & $\{r+1,2 r-1\}$ \\
$\{r+1,2 r+1,2 r-1\}$ & $x_{1}$ & $\{r+1,2 r+1\}$ \\
$\vdots$ & $\vdots$ & $\vdots$ \\
$\{r+1,2 r+1,2 r-1+(r-3)\}$ & $x_{1}$ & $\{r+1,2 r+1\}$ \\
$\exp \left(\mathscr{A}(G(r, r, 4))^{\prime \prime}, \kappa\right)=\{r+1,2 r+1,3(r-1)\}$ & & \\
\hline
\end{tabular}

TABle 3. Induction Table for $\left(\mathscr{A}(G(r, r, 4))^{\prime \prime}, \kappa\right)$.

Next let $W$ be of type $G_{29}$. Since $W$ is transitive on $\mathscr{A}\left(G_{29}\right)$, we may take $H_{0}=\operatorname{ker} x_{4}$. Then the defining polynomial for $\left(\mathscr{A}\left(G_{29}\right)^{\prime \prime}, \kappa\right)$ is given by

$$
\begin{aligned}
Q\left(\mathscr{A}\left(G_{29}\right)^{\prime \prime}, \kappa\right)= & \left(x_{1}-x_{2}-i x_{3}\right)^{2}\left(x_{1}-x_{2}\right)\left(x_{2}-x_{3}\right) x_{3}^{3}\left(x_{1}-i x_{2}-x_{3}\right)^{2}\left(x_{1}-x_{2}+i x_{3}\right)^{2} \\
& \left(x_{1}-x_{3}\right)\left(x_{1}+i x_{2}-i x_{3}\right)^{2} x_{2}^{3}\left(x_{1}+i x_{2}-x_{3}\right)^{2}\left(x_{1}-i x_{2}+i x_{3}\right)^{2} \\
& x_{1}^{3}\left(x_{1}-i x_{2}+x_{3}\right)^{2}\left(x_{1}-i x_{2}-i x_{3}\right)^{2}\left(x_{1}+i x_{2}+i x_{3}\right)^{2}\left(x_{1}+i x_{2}+x_{3}\right)^{2} \\
& \left(x_{1}+x_{2}-i x_{3}\right)^{2}\left(x_{1}+x_{2}+i x_{3}\right)^{2}\left(x_{2}+x_{3}\right)\left(x_{1}+x_{3}\right)\left(x_{1}+x_{2}\right) .
\end{aligned}
$$

We present an induction table for $\left(\mathscr{A}\left(G_{29}\right)^{\prime \prime}, \kappa\right)$ in Table 4 . Note that since $\mathscr{A}\left(G_{29}\right)^{\prime \prime}$ is of rank 3 each of the restrictions in Table 4 is of rank 2 and so is inductively free. 


\begin{tabular}{lll}
\hline $\exp \left(\mathscr{A}^{\prime}, \mu^{\prime}\right)$ & $\alpha_{H}$ & $\exp \left(\mathscr{A}^{\prime \prime}, \mu^{*}\right), \mu^{*}$ \\
\hline \hline $\exp \mathscr{A}\left(G_{29}\right)^{\prime \prime}=\{1,9,11\}$ & $x_{1}-x_{2}-i x_{3}$ & $\{9,11\},(1,2,2,4,2,3,4,2)$ \\
$\{2,9,11\}$ & $x_{3}$ & $\{9,11\},(3,4,3,4,3,3)$ \\
$\{3,9,11\}$ & $x_{3}$ & $\{9,11\},(3,4,3,4,3,3)$ \\
$\{4,9,11\}$ & $x_{1}-i x_{2}-x_{3}$ & $\{9,11\},(2,1,2,2,4,3,4,2)$ \\
$\{5,9,11\}$ & $x_{1}-x_{2}+i x_{3}$ & $\{9,11\},(1,2,2,3,2,4,4,2)$ \\
$\{6,9,11\}$ & $x_{1}+i x_{2}-i x_{3}$ & $\{9,11\},(2,2,3,2,1,4,2,4)$ \\
$\{7,9,11\}$ & $x_{2}$ & $\{9,11\},(3,4,3,4,3,3)$ \\
$\{8,9,11\}$ & $x_{2}$ & $\{9,11\},(3,4,3,4,3,3)$ \\
$\{9,9,11\}$ & $x_{1}+i x_{2}-x_{3}$ & $\{9,11\},(2,2,2,3,2,1,4,4)$ \\
$\{9,10,11\}$ & $x_{1}-i x_{2}+i x_{3}$ & $\{9,11\},(2,2,3,2,1,4,2,4)$ \\
$\{9,11,11\}$ & $x_{1}$ & $\{9,11\},(3,3,3,3,4,4)$ \\
$\{9,11,12\}$ & $x_{1}$ & $\{9,11\},(3,3,3,3,4,4)$ \\
$\{9,11,13\}$ & $x_{1}-i x_{2}+x_{3}$ & $\{9,13\},(2,2,2,5,1,2,3,5)$ \\
$\{9,12,13\}$ & $x_{1}-i x_{2}-i x_{3}$ & $\{9,13\},(2,1,2,5,3,5,2,2)$ \\
$\{9,13,13\}$ & $x_{1}+i x_{2}+i x_{3}$ & $\{9,13\},(2,1,2,5,3,5,2,2)$ \\
$\{9,13,14\}$ & $x_{1}+i x_{2}+x_{3}$ & $\{9,13\},(2,5,2,5,2,3,1,2)$ \\
$\{9,13,15\}$ & $x_{1}+x_{2}-i x_{3}$ & $\{9,13\},(2,2,3,1,2,5,5,2)$ \\
$\{9,13,16\}$ & $x_{1}+x_{2}+i x_{3}$ & $\{9,13\},(1,2,2,5,2,3,5,2)$ \\
$\exp \left(\mathscr{A}\left(G_{29}\right)^{\prime \prime}, \kappa\right)=\{9,13,17\}$ & & \\
\hline & & \\
\hline & & \\
\hline & &
\end{tabular}

TABLE 4. Induction Table for $\left(\mathscr{A}\left(G_{29}\right)^{\prime \prime}, \kappa\right)$.

By the data in Tables 3, 4 and Remark 2.19, $\left(\mathscr{A}^{\prime \prime}, \kappa\right)$ is inductively free in each instance.

We mention that it follows from Theorem 2.3 and Lemmas 4.8 and 4.9 that the implication in Corollary 2.20 fails without the assumption that $\mathscr{A}^{\prime \prime}$ is inductively free.

Lemma 4.10. Let $W$ be of type $G_{33}$ or $G_{34}$. Then $\left(\mathscr{A}(W)^{\prime \prime}, \kappa\right)$ is not inductively free.

Proof. First consider the case of $W=G_{33}$. Let $\mathscr{A}=\mathscr{A}(W)$. Direct computations have shown that there is no chain of free multiplicities from the zero multiplicity on $\mathscr{A}^{\prime \prime}$ to $\kappa$. The maximal length of such chains is $22<44=|\kappa|$. Hence $\left(\mathscr{A}^{\prime \prime}, \kappa\right)$ is not inductively free.

Finally, let $W=G_{34}$ and $\mathscr{A}=\mathscr{A}(W)$. Then $W$ admits a parabolic subgroup $W_{X}$ of type $G(3,3,5)$, by [OT92, Table C.15]. Thanks to [OT92, Cor. 6.28], we have $\mathscr{A}\left(W_{X}\right)=$ $\mathscr{A}(W)_{X}=\mathscr{A}_{X}$. Fix $H_{0} \in \mathscr{A}_{X}$. Then considering the restriction with respect to $H_{0}$, we have $\left(\mathscr{A}^{\prime \prime}\right)_{X}=\left(\mathscr{A}_{X}\right)^{\prime \prime}$. So we see that $\left(\left(\mathscr{A}^{\prime \prime}\right)_{X}, \kappa_{X}\right)=\left(\left(\mathscr{A}_{X}\right)^{\prime \prime}, \kappa_{X}\right)=\left(\mathscr{A}(G(3,3,5))^{\prime \prime}, \kappa\right)$, by Lemma 2.16(ii). According to Lemma 4.7, the latter is not inductively free, thus neither is $\left(\mathscr{A}\left(G_{34}\right)^{\prime \prime}, \kappa\right)$, owing to Theorem 2.22.

Finally, Theorems 1.4 and 1.9 follow from the results from $\S 4.1$ to $\S 4.5$ above. 
Remark 4.11. The fact that $\left(\mathscr{A}\left(G_{33}\right)^{\prime \prime}, \kappa\right)$ is not inductively free, proved in Lemma 4.10, and the data in the induction tables 2 and 4 were obtained by computational means. Specifically, we retrieved relevant data about reflection groups from some GAP code provided by J. Michel [M15]; see also [S+97] and [GHL ${ }^{+96}$. Subsequently, we made use of SAGE, cf. [Ste15], and SINGULAR, cf. [GPS09], in order to calculate the various bases of modules of derivations involved.

Acknowledgments: We acknowledge support from the DFG-priority program SPP1489 "Algorithmic and Experimental Methods in Algebra, Geometry, and Number Theory". Part of the research for this paper was carried out while the authors were staying at the Mathematical Research Institute Oberwolfach supported by the "Research in Pairs" programme.

\section{REFERENCES}

[ATW08] T. Abe, H. Terao, and M. Wakefield, The Euler multiplicity and addition-deletion theorems for multiarrangements. J. Lond. Math. Soc. (2) 77 (2008), no. 2, 335-348.

[AY13] T. Abe and M. Yoshinaga, Free arrangements and coefficients of characteristic polynomials, Math. Z. 275 (2013), no. 3-4, 911-919.

[AHR14] N. Amend, T. Hoge and G. Röhrle, On inductively free restrictions of reflection arrangements, J. Algebra 418 (2014), 197-212.

[BC12] M. Barakat and M. Cuntz, Coxeter and crystallographic arrangements are inductively free, Adv. Math 229 (2012), 691-709.

[CR16] H. Conrad and G. Röhrle, On inductively free multiderivations of braid arrangements, Ann. Comb., 20, (2016), 719-735

[CRS17] M. Cuntz, G. Röhrle, and A. Schauenburg, Arrangements of ideal type are inductively free, https://arxiv.org/abs/1711.09760

[GHL $\left.{ }^{+} 96\right]$ M. Geck, G. Hiß, F. Lübeck, G. Malle, and G. Pfeiffer, CHEVIE - A system for computing and processing generic character tables, Appl. Algebra Engrg. Comm. Comput. 7 (1996), 175-210.

[GPS09] G.-M. Greuel, G. Pfister, and H. Schönemann, Singular 3-1-1, A Computer Algebra System for Polynomial Computations, Centre for Computer Algebra, University of Kaiserslautern, 2009.

[HR15] T. Hoge and G. Röhrle, On inductively free reflection arrangements, J. Reine u. Angew. Math. 701 (2015), 205-220.

[HRS17] T. Hoge, G. Röhrle and A. Schauenburg, Inductive and Recursive Freeness of Localizations of multiarrangements, in: Algorithmic and Experimental Methods in Algebra, Geometry, and Number Theory, Springer Verlag 2017.

[M15] J. Michel, The development version of the CHEVIE package of GAP3. J. Algebra, 435 (2015), $308-336$.

[OS82] P. Orlik and L. Solomon, Arrangements Defined by Unitary Reflection Groups, Math. Ann. 261, (1982), 339-357.

[OT92] P. Orlik and H. Terao, Arrangements of hyperplanes, Springer-Verlag, 1992.

[S $\left.{ }^{+} 97\right] \quad M$. Schönert et al., GAP - Groups, Algorithms, and Programming - version 3 release 4, 1997.

[ST54] G.C. Shephard and J.A. Todd, Finite unitary reflection groups. Canadian J. Math. 6, (1954), 274-304.

[S12] M. Schulze, Freeness and multirestriction of hyperplane arrangements. Compos. Math. 148 (2012), no. 3, 799-806.

[Ste15] W. A. Stein, et al, Sage Mathematics Software (Version 7.5.1), The Sage Development Team, 2015, http://www. sagemath.org.

[Ste60] R. Steinberg, Invariants of finite reflection groups, Canad. J. Math. 12, (1960), 616-618.

[T80a] H. Terao, Arrangements of hyperplanes and their freeness I, II, J. Fac. Sci. Univ. Tokyo 27 (1980), 293-320. 
[T80b] - Free arrangements of hyperplanes and unitary reflection groups. Proc. Japan Acad. Ser. A Math. Sci. 56 (1980), no. 8, 389-392.

[Y04] M. Yoshinaga, Characterization of a free arrangement and conjecture of Edelman and Reiner. Invent. Math. 157 (2004), no. 2, 449-454.

[Y05] _ On the freeness of 3-arrangements. Bull. London Math. Soc. 37 (2005), no. 1, 126-134.

[Y14] _ Freeness of hyperplane arrangements and related topics, Ann. Fac. Sci. Toulouse Math. (6) 23 (2014), no. 2, 483-512.

[Z89] G. Ziegler, Multiarrangements of hyperplanes and their freeness. Singularities (Iowa City, IA, 1986), 345-359, Contemp. Math., 90, Amer. Math. Soc., Providence, RI, 1989.

Institut für Algebra, Zahlentheorie und Diskrete Mathematik, Fakultät für Mathematik und Physik, Leibniz Universität Hannover, Welfengarten 1, 30167 Hannover, Germany

E-mail address: hoge@math.uni-hannover.de

Fakultät für Mathematik, Ruhr-Universität Bochum, D-44780 Bochum, Germany

E-mail address: gerhard.roehrle@rub.de 